\title{
Elucidating the germanium distribution in ITQ-13 zeolites by density functional theory
}

\author{
Michael Fischer ${ }^{1,2,3^{*}}$, Carlos Bornes ${ }^{4}$, Luís Mafra ${ }^{4}$, João Rocha ${ }^{4}$
}

1) Faculty of Geosciences, University of Bremen, Klagenfurter Straße 2-4, 28359 Bremen, Germany

2) Bremen Center for Computational Materials Science (BCCMS), University of Bremen, 28359 Bremen, Germany

3) MAPEX Center for Materials and Processes, University of Bremen, 28359 Bremen, Germany

4) CICECO, Aveiro Institute of Materials, Department of Chemistry, University of Aveiro, 3810-193 Aveiro, Portugal

Corresponding author: michael.fischer@uni-bremen.de

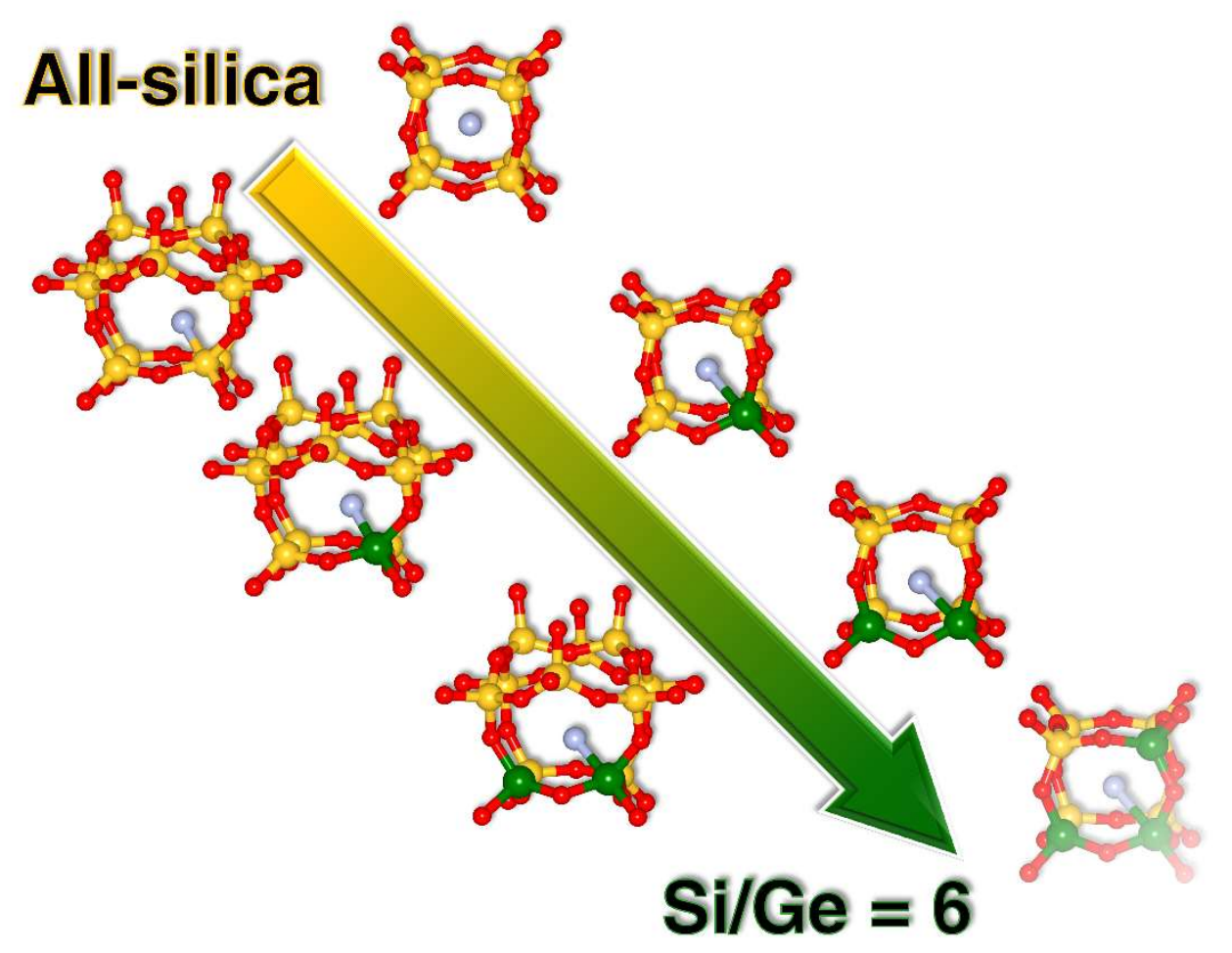




\section{Abstract}

ITQ-13 is a medium-pore zeolite that can be prepared in all-silica form and as silicogermanate with $\mathrm{Si} / \mathrm{Ge}$ ratios as low as 3. Usually synthesised in the presence of fluoride, ITQ-13 is among the very few systems containing fluoride anions in two distinct cage types, cube-like $d 4 r$ units and $\left[4 \cdot 5^{6}\right]$ cages. Here, dispersion-corrected density functional theory (DFT) calculations are used to investigate the energetically most favourable Ge distributions for Si/Ge ratios between 55 and 6 . The calculations show Ge atoms are incorporated at both the corners of $d 4 r$ cages and at the basal plane of the $\left[4 \cdot 5^{6}\right]$ cages, in accordance with ${ }^{19} \mathrm{~F}-\mathrm{NMR}$ spectroscopy. Two Ge atoms at adjacent corners of $\left[4 \cdot 5^{6}\right]$ cages are stable at the highest $\mathrm{Ge}$ content considered $(\mathrm{Si} / \mathrm{Ge}=6)$. Such a local environment has not yet been considered in the experimental literature. A calculation of the corresponding ${ }^{19} \mathrm{~F}-\mathrm{NMR}$ resonance points to overlap with other resonances, which might preclude its clear identification. Additional calculations investigate the variation of the dynamic behaviour of the fluoride anions as a function of the local environment as well as the selective defluorination of the $\left[4 \cdot 5^{6}\right]$ cages. 


\section{Introduction}

The zeolite ITQ-13 was first reported by Corma's group from the Institute of Chemical Technology (Instituto de Tecnología Química, ITQ) at the Polytechnic University of Valencia in a patent published in 2002. ${ }^{[1]}$ ITQ-13 is a medium-pore zeolite with a three-dimensional pore system consisting of channels formed by nine- and ten-membered rings (9MR/10MR) of $\mathrm{TO}_{4}$ tetrahedra. ${ }^{[2]}$ In addition to the all-silica form, labelled ITQ-13_SiO 2 throughout this article, this zeolite can also be synthesised as borosilicate, aluminosilicate, ${ }^{[2,3]}$ and silicogermanate. ${ }^{[1,4]}$ Due to its narrow channel system and high acidity, ITQ-13 is a very interesting material for catalytic applications. In the cracking of vacuum gasoil, Castañeda et al. observed a higher propene/propane ratio when employing ITQ-13 instead of the widely used zeolite ZSM-5 as catalyst additive. ${ }^{[3]}$ They attributed this difference to a higher shape selectivity towards propene caused by the narrower pore dimensions, a finding that was confirmed in subsequent work. ${ }^{[5]}$ ITQ-13 was also found to give a product spectrum different from other zeolite catalysts (ZSM-5, MCM-22) in methanol-to-hydrocarbon (MTH) conversions, potentially enhancing the flexibility of the MTH process in the context of varying demands for different products. ${ }^{[6]}$

ITQ-13 is the type material of the ITH framework, ${ }^{[7]}$ which can be decomposed into four types of discrete cages: double-four ring $(d 4 r)$ cages (face symbol $\left[4^{6}\right]$, labelled t-cub in the nomenclature of natural tilings ${ }^{[8]}$ ), lau cages (face symbol $\left[4^{2} \cdot 6^{4}\right]$, t-lau), st $f$ cages (face symbol $\left[4 \cdot 5^{6}\right]$, t-nuh) and $\left[4 \cdot 5^{2} \cdot 6^{2}\right]$ cages (t-mel). It may be noted that the face symbol $\left[4 \cdot 5^{2} \cdot 6^{2}\right]$ was used for the st $f$ cages in various earlier works. ${ }^{[2,4,9-13]}$ However, the 6MRs are not strong rings, being the sum of two 5MRs, and therefore this symbol does not conform to the rules outlined in natural tiling theory. ${ }^{[8]}$ The arrangement of $2 d 4 r$ cages, 2 stf cages, 4 lau cages, and $6\left[4 \cdot 5^{2} \cdot 6^{2}\right]$ cages in the unit cell (u.c.) results in the formation of $9 M R$ channels running along the crystallographic $a$ direction, and 10MR channels running along $b$ and c (Figure 1). In the as-synthesised form of all-silica ITQ-13, the head groups of the hexamethonium dications that are used as organic structure-directing agents (OSDAs) are located at the intersection of 9MR and 10MR channels, with the alkyl chain oriented parallel to $c$. The synthesis of ITQ-13_SiO requires both use of an OSDA and the addition of fluoride to the reaction mixture, as the fluoride anions play a structure directing role, particularly in the formation of the $d 4 r$ cages. The crystal structure determination showed that these anions reside in two different environments: ${ }^{[2]}$ One half of the fluoride anions occupies the centre of the $d 4 r$ cages, whereas the other half is bonded to an $\mathrm{Si}$ atom at the basal $4 \mathrm{MR}$ plane of the $\left[4 \cdot 5^{6}\right]$ cages, forming an $\left[\mathrm{SiO}_{4} \mathrm{~F}\right]^{-}$trigonal bipyramid. The latter fluoride location is disordered over four symmetry-equivalent positions in the cage with an occupancy of 0.25 . The coexistence of two different fluoride environments in one zeolite structure is a relatively rare phenomenon, although another example has been reported recently for the case of all-silica MWW. ${ }^{[14]}$ 
a)
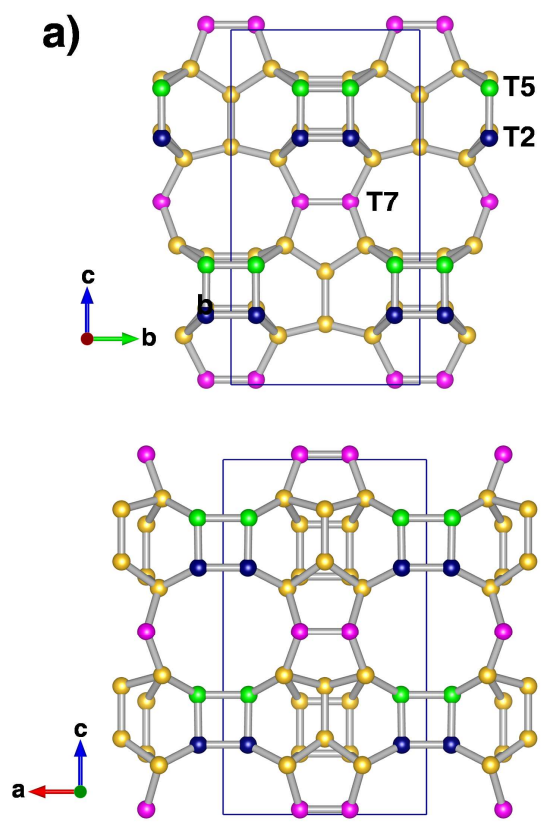

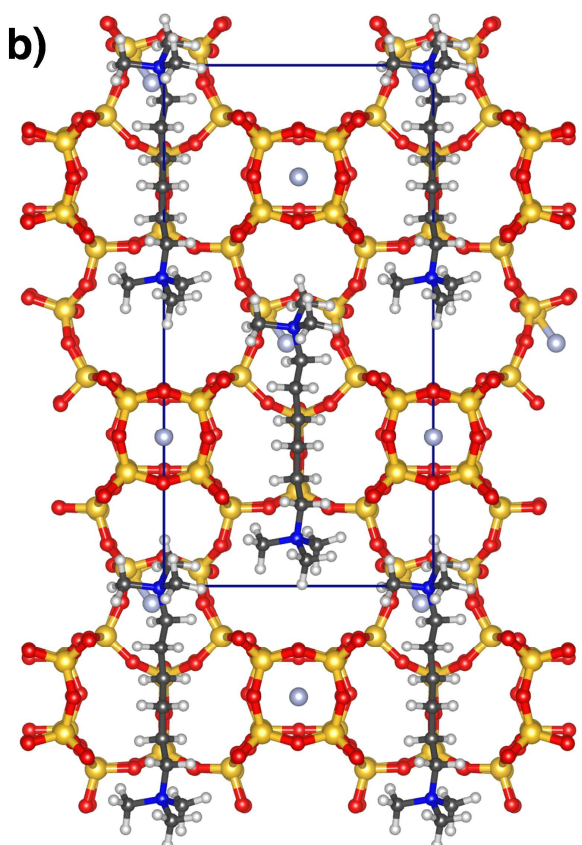

Figure 1: a) Skeletal representation of the ITH framework, shown in projections along [100] (top) and [010] (bottom). Crystallographically distinct $\mathrm{T}$ sites that are preferentially occupied by Ge in Gecontaining ITQ-13 are labelled and shown in blue (T2), green (T5), and pink (T7), other T sites are shown in yellow. b) Atomistic representation of the fully ordered ITQ-13_SiO 2 model used in the calculations, projection along [100]. Colour scheme: Yellow $=\mathrm{Si}$, red $=$ oxygen, pale blue $=$ fluorine, grey $=$ carbon, blue $=$ nitrogen, light grey $=$ hydrogen .

Due to the sensitivity of the ${ }^{19} \mathrm{~F}-\mathrm{NMR}$ chemical shift to the local environment, solid-state NMR can help to elucidate which $T$ atoms are found at the corners of the cages that host the fluoride anions. This is particularly interesting for silicogermanates, as X-ray diffraction methods cannot provide insights into the local structure due to lack of long-range order in the Ge distribution. In-depth studies of ITQ-13 samples containing different amounts of Ge have been reported by Vidal-Moya et al. ${ }^{[4]}$ and by Liu et al. ${ }^{[11,12]}$ The ITQ-13_ $\mathrm{SiO}_{2}{ }^{19} \mathrm{~F}$ resonance at $\delta=-38 \mathrm{ppm}$ was ascribed to fluoride anions encapsulated in $d 4 r$ cages, ${ }^{[15]}$ whereas the peak at $\delta=-66$ ppm stems from $\left[\mathrm{SiO}_{4} \mathrm{~F}\right]^{-}$trigonal bipyramids. ${ }^{[16]}$ It is known from the crystal structure that these bipyramids are part of the basal plane of the $\left[4 \cdot 5^{6}\right]$ cages. ${ }^{[2]}$ The incorporation of Ge gives rise to additional resonances at $\delta=-8 \mathrm{ppm},-20 \mathrm{ppm}$, and $-55 \mathrm{ppm}$. These peaks vary in intensity depending on the Si/Ge ratio, whereas those at $\delta=-38$ and $-66 \mathrm{ppm}$ decrease and, ultimately, disappear upon increasing Ge content. The intensity evolution of the five distinct ${ }^{19} \mathrm{~F}$ NMR resonances observed by Vidal-Moya et al. ${ }^{[4]}$ and by Liu et al. ${ }^{[11]}$ is summarised in Table 1. Although the resonance at $\delta=-55 \mathrm{ppm}$ was straightforwardly attributed to fluoride in $\left[4 \cdot 5^{6}\right]$ cages having one Ge atom in the basal $4 \mathrm{MR}^{, 4]}$ the assignment of the remaining two resonances has been the matter of some debate, and $d 4 r$ cages with different amounts and/or arrangements of Si and Ge were considered to be responsible for their appearance..$^{[12,17-19]}$ Recent, combined experimental and computational 
studies of ITQ-13 ${ }^{[12]}$ and STW-type silicogermanates ${ }^{[19]}$ agree that the resonance at $\delta=-20$ ppm can be assigned to $d 4 r$ cages with isolated Ge atoms (i.e., Ge atoms having only Si atoms at neighbouring corners of the cage), whereas the resonance at $\delta=-8 \mathrm{ppm}$ is due to fluoride in $d 4 r$ cages having at least one Ge-O-Ge link. Configurations with different numbers of isolated Ge atoms or different numbers of Ge-O-Ge links give rise to ${ }^{19} \mathrm{~F}-\mathrm{NMR}$ resonances with very similar chemical shifts, requiring $2 \mathrm{D}{ }^{19} \mathrm{~F}$ ${ }^{29} \mathrm{Si} \mathrm{NMR} \mathrm{correlation} \mathrm{experiments} \mathrm{to} \mathrm{(partially)} \mathrm{resolve} \mathrm{them;}{ }^{[12]}$ as a consequence, there are only two broad peaks in the 1D NMR spectra, despite a multitude of possible local arrangements. ${ }^{[4,11]}$

Table 1: Intensity of distinguishable ${ }^{19} \mathrm{~F}-\mathrm{NMR}$ resonances of ITQ-13 samples with different Ge contents. ${ }^{[4,11]}$ The assignment to different building units according to more recent studies is given in the column header. Intensities are given on a qualitative scale, based on the NMR spectra shown in the original articles.

\begin{tabular}{|c|c|c|c|c|c|}
\hline$\delta_{\text {exp }} / \mathrm{ppm}$ & -8 & -20 & -38 & -55 & (-66 \\
\hline Building unit & $\begin{array}{l}d 4 r \text { with at } \\
\text { least one } \\
\text { Ge-O-Ge link }\end{array}$ & $\begin{array}{l}d 4 r \text { with } \\
\text { only isolated } \\
\text { Ge atoms }\end{array}$ & $\mathrm{SiO}_{2}-d 4 r$ & {$\left[4 \cdot 5^{6}\right]_{1 \mathrm{Ge}}$} & $\mathrm{SiO}_{2}-\left[4 \cdot 5^{6}\right]$ \\
\hline ITQ-13_SiO ${ }^{[4,11]}$ & Absent & Absent & Very strong & Absent & Medium \\
\hline $\begin{array}{l}\text { ITQ-13, } \mathrm{Si} / \mathrm{Ge}=25^{[4]} \\
(\approx 2.2 \mathrm{Ge} / \text { u.c. })\end{array}$ & Weak & Strong & Strong & Weak & Strong \\
\hline $\begin{array}{l}\text { ITQ-13, } \mathrm{Si} / \mathrm{Ge}=21^{[11]} \\
(\approx 2.5 \mathrm{Ge} / \text { u.c. })\end{array}$ & Weak & Medium & Strong & Very weak & Strong \\
\hline $\begin{array}{l}\text { ITQ-13, } \mathrm{Si} / \mathrm{Ge}=11^{[4]} \\
(\approx 4.7 \mathrm{Ge} / \text { u.c. })\end{array}$ & Strong, broad & Strong & Very weak & Weak & Weak \\
\hline $\begin{array}{l}\text { ITQ-13, Si/Ge }=9^{[11]} \\
\text { ( } \approx 5.6 \mathrm{Ge} / \text { u.c. })\end{array}$ & Strong, broad & Medium & Very weak & Not shown & Not shown \\
\hline $\begin{array}{l}\text { ITQ }-13, \mathrm{Si} / \mathrm{Ge}=6^{[4]} \\
(\approx 8.0 \mathrm{Ge} / \text { u.c. })\end{array}$ & Strong, broad & Weak & Absent & Weak & Very weak \\
\hline $\begin{array}{l}\text { ITQ-13, } \mathrm{Si} / \mathrm{Ge}=4.5^{[11]} \\
(\approx 10.2 \mathrm{Ge} / \text { u.c. })\end{array}$ & Strong, broad & Weak & Absent & Not shown & Not shown \\
\hline $\begin{array}{l}\text { ITQ-13, } \mathrm{Si} / \mathrm{Ge}=3.2^{[11]} \\
(\approx 13.3 \mathrm{Ge} / \text { u.c. })\end{array}$ & Strong, broad & Absent & Absent & Not shown & Not shown \\
\hline
\end{tabular}

The analysis of the ${ }^{19} \mathrm{~F}$ - and ${ }^{29} \mathrm{Si}-\mathrm{NMR}$ spectra of ITQ-13 samples with different Ge contents resulted in the conclusion that Ge atoms preferentially occupy the T2 and T5 sites, which are associated with the $d 4 r$ cages, and the T7 sites, which form the basal plane of the $\left[4 \cdot 5^{6}\right]$ cages. ${ }^{[4]}$ It is important to note that the $d 4 r$ cages form the sole connection of layers constituted by the other building units along the $a$ direction (Figure 1). As an acid treatment causes a selective breaking of $\mathrm{Ge}-\mathrm{O}$ bonds, the $d 4 r$ cages in 
Ge-rich ITQ-13 samples can be removed, resulting in layered solids that could be used as precursors in the synthesis of other mesostructured materials by means of the Assembly-Disassembly-OrganisationReassembly (ADOR) process. ${ }^{[13]}$ Furthermore, the (partial) acid leaching degermanation of ITQ-13 samples through acid leaching results in the formation of mesopores, which can improve the accessibility of the active sites and, hence, increase the catalytic activity. ${ }^{[2,21]}$

The presence of Ge atoms in the vicinity of the fluoride anions also influences the fluoride removal behaviour. In the all-silica zeolite ITQ-13_SiO ${ }_{2}$, an alkaline treatment of the as-synthesised sample at elevated temperature resulted in a complete removal of fluoride from the $\left[4 \cdot 5^{6}\right]$ cages, whereas fluoride anions occupying the $d 4 r$ cages remained in the structure. ${ }^{[10]}$ The crystallinity of the zeolite was fully retained. In Ge-containing samples, fluoride could also be removed from the $d 4 r$ cages, but the degree of fluoride removal depended heavily on the Ge content. ${ }^{[11]}$ This indicates that the number of Ge atoms at the corners of the $d 4 r$ cages, and possibly also their local arrangement with respect to each other, strongly influence the thermodynamics and/or kinetics of defluorination.

Since experimental investigations provide only indirect insights, computational methods are an important alternative pathway to predict the preferred Ge locations and/or respective arrangements of Ge atoms in a given zeolite structure. Both force field methods ${ }^{[18,22,23]}$ and density functional theory (DFT) calculations ${ }^{[24-28]}$ have been used for this purpose, addressing silicogermanate zeolites with topologies of varying complexity. Since the number of possible configurations quickly becomes very large when introducing several Ge atoms per unit cell (an issue that will be discussed in more detail for the specific case of ITQ-13 in the Results section), a comprehensive sampling is usually unfeasible and findings obtained for low Ge contents were typically extrapolated to higher Ge contents to generate a limited set of probable configurations. Altogether, the computational investigations corroborated the preference of Ge to locate in $d 4 r$ units, as well as an increased stability of arrangements containing Ge-O-Ge links.

In this work, dispersion-corrected DFT calculations are performed to investigate the energetically preferred Ge distributions in ITQ-13 zeolites having Si/Ge ratios between 55 and 6, considering realistic models of as-synthesised zeolites that include fluoride anions and OSDA cations. For Ge contents corresponding to more than one Ge atom per unit cell, a variety of different configurations are sampled, considering, however, only an occupation of the T2, T5, and T7 sites. At low Si/Ge ratios (= high Ge contents), calculations predict the stability of $\left[4 \cdot 5^{6}\right]$ cages with two Ge atoms in the 4MR. As such cages have not yet been considered in experimental studies, ${ }^{19} \mathrm{~F}$ chemical shift calculations are performed to identify the resonance of the fluoride anions incorporated in these building units. For selected ITQ-13 models, DFT-based molecular dynamics calculations are carried out to investigate how the dynamic behaviour of the fluoride anions is affected by the local environment. Finally, the 
thermodynamics of fluoride removal are studied using calculations for partially defluorinated ITQ-13 models.

\section{Computational details}

\section{ITQ-13 models}

The crystal structure of as-synthesised ITQ-13_SiO 2 (orthorhombic, space group Amm2) was taken as starting point. ${ }^{[2]}$ The disorder of the hexamethonium OSDAs and of the fluoride anions incorporated in the $\left[4 \cdot 5^{6}\right]$ cages was removed, resulting in a fully ordered structure that is depicted in Figure $\mathbf{1} \mathbf{~ b}$. The removal of disorder inevitably reduces the symmetry, and only the $A$-centering is retained in the final structure model of ITQ-13_SiO 2 . This model was optimised using the dispersion-corrected DFT approach described in the following subsection, optimising all atomic coordinates and the unit cell parameters $a, b$, and $c$, but fixing the unit cell angles to 90 degrees. Models of ITQ-13 containing 1, 2, $3,4,6$, or $8 \mathrm{Ge}$ atoms per (conventional) unit cell were constructed on the basis of the DFT-optimised structure of ITQ-13_SiO 2 . Again, the atomic coordinates and $a, b$, and $c$ were optimised, fixing the angles to 90 degrees. For partially defluorinated models, different approaches were compared (described in detail in the corresponding part of the Results section).

\section{Periodic DFT calculations and DFT-based molecular dynamics simulations}

DFT structure optimisations and DFT-based ab-initio molecular dynamics (AIMD) simulations were carried out using the CP2K code, ${ }^{[29]}$ version 7.1, installed on the HLRN-IV facilities (HLRN - NorthGerman Supercomputing Alliance). The Quickstep DFT module integrated into CP2K is based on a mixed Gaussian and plane wave approach. ${ }^{[30]}$ All calculations used the PBE exchange-correlation functional ${ }^{[31]}$ and the D3 dispersion correction proposed by Grimme et al. ${ }^{[32]} \mathrm{A}$ plane wave cutoff energy of 600 Ry was employed, and the first Brillouin zone was sampled at the $\ulcorner$ point, only. The structure optimisations used "molecularly optimised" triple-zeta (TZVP-MOLOPT) basis sets, whereas the AIMD simulations made use of double-zeta (DZVP-MOLOPT-SR) basis sets. ${ }^{[33]}$ The core electrons were represented with Goedeker-Teter-Hutter pseudopotentials devised by Krack. ${ }^{[34]}$ The structure optimisations were considered converged when the maximal residual force was smaller than $10^{-6} \mathrm{Ha}$ bohr ${ }^{-1}$ and the maximum geometry change was below $2 \cdot 10^{-5}$ bohr. The pressure tolerance in the variable-cell optimisations was set to $5 \cdot 10^{-4} \mathrm{GPa}$. The AIMD simulations were carried out for two temperatures, $298 \mathrm{~K}$ and $408 \mathrm{~K}$, in the canonical (NVT) ensemble. Using a time step of $0.5 \mathrm{fs}$, the simulations were equilibrated for 10,000 steps ( 5 ps), followed by a production stage of 30,000 steps 
(15 ps). A Nosé-Hoover thermostat was used with a time constant of 50 ps. ${ }^{[35,36]}$ For each system, three independent trajectories were computed for each temperature. From the AIMD trajectories, root mean square displacements (RMSDs) of fluoride anions were computed using the VMD software. ${ }^{[37]}$ For cases of particular interest, the evolution of the atomic coordinates of individual fluoride anions was evaluated. AIMD average structures, in which the coordinates of the constituent atoms were obtained by averaging over the instantaneous positions during the production phase of the AIMD simulation, were also computed with VMD. All structure visualisations were prepared using VESTA. ${ }^{[38]}$

\section{Cluster DFT calculations of the isotropic ${ }^{19} \mathrm{~F}$ shifts}

To obtain ${ }^{19} \mathrm{~F}$ chemical shifts associated with different fluoride-containing building units, non-periodic cluster models of the relevant building units were extracted from the DFT-optimised structures, and saturated by attaching hydrogen atoms to the "dangling" oxygen atoms. ${ }^{19} \mathrm{~F}$ isotropic chemical shifts were computed using the gauge-independent atomic orbital method ${ }^{[39]}$ as implemented in Gaussian 16 revision C.01. ${ }^{[40]}$ Two hybrid exchange-correlation functionals, $\mathrm{PBEO}^{[41]}$ and B3PW91, ${ }^{[42,43]}$ were tested with Dunning's correlation consistent triple-zeta basis sets (cc-pVTZ). ${ }^{[44,45]}$ The B3PW91 functional has been employed in previous computational predictions of the ${ }^{19} \mathrm{~F}$ chemical shifts in fluoride-containing silicogermanates, ${ }^{[12,18]}$ whereas the PBEO functional was used with some success for other fluorine-bearing species. ${ }^{[46]}$ In each case, the computed chemical shifts were referenced against the ${ }^{19} \mathrm{~F}$ chemical shift of $\mathrm{CCl}_{3} \mathrm{~F}(\delta=0 \mathrm{ppm})$ obtained with the same method.

\section{Results and discussion}

\section{Preferred Ge distributions}

All-silica ITQ-13: To begin with, the structure of purely siliceous ITQ-13 was optimised after removing the disorder of fluoride anions and hexamethonium OSDAs as described above. The resulting unit cell parameters, $a=12.607 \AA, b=11.467 \AA, c=22.188 \AA$, are only slightly overestimated when compared to the experimental values ( $a=12.525 \AA, b=11.391 \AA, c=22.053 \AA$ ). This confirms the previous finding that the PBE-D3 functional delivers excellent predictions of the unit cell parameters of all-silica zeolites. ${ }^{[47,48]}$ The $\mathrm{F} 1$ atom resides at the centre of the $d 4 r$ cage, with all eight F-Si distances falling between 2.6 and $2.7 \AA$ (Figure 2). As in the starting structure, the F2 atom is bonded to one of the Si7 
atoms, with an F2-Si7 distance of $1.78 \AA$ (Figure 2). This distance agrees well with previous NMR and DFT results for zeolites containing trigonal-bipyramidal $\mathrm{SiO}_{4} \mathrm{~F}^{-}$units. ${ }^{[48-50]}$

\section{d4r cage}
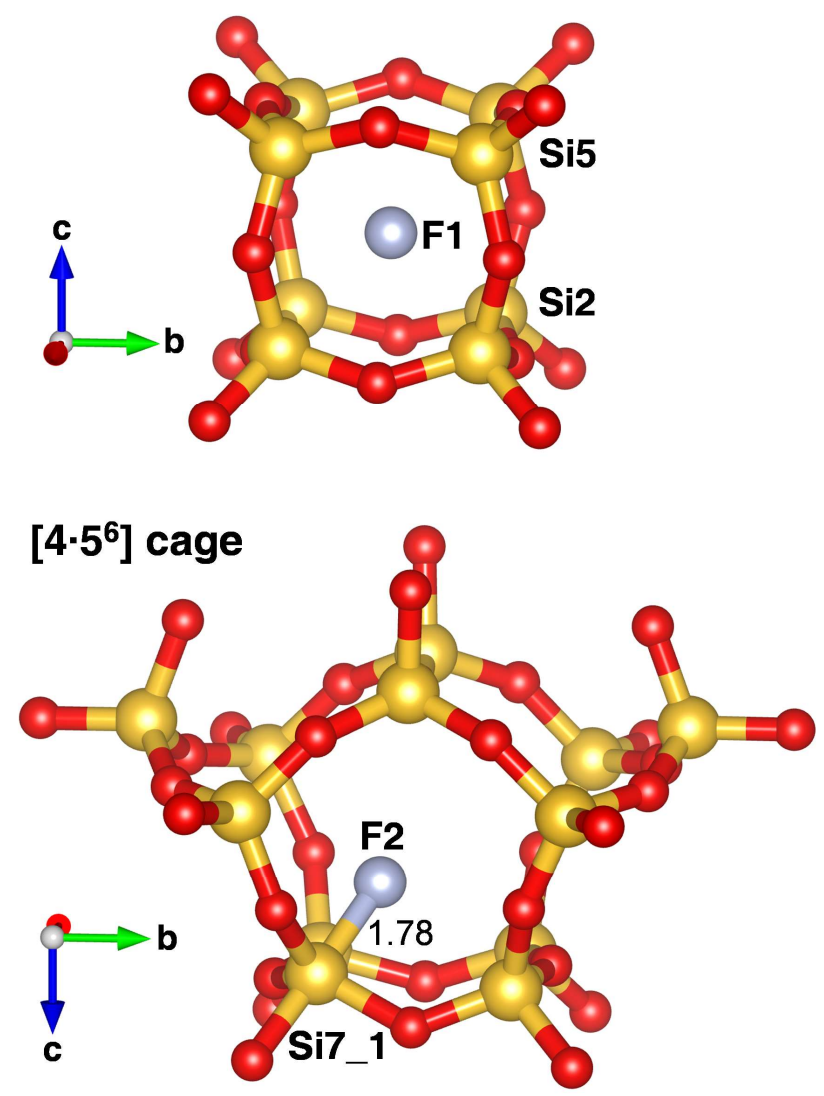

Figure 2: Fluoride environments in DFT-optimised structure of ITQ-13_SiO ${ }_{2}$. The length of the F-Si bond in the $\left[\mathrm{SiO}_{4} \mathrm{~F}\right]^{-}$unit is given in $\AA$.

$1 \mathrm{Ge}$ atom per unit cell $(\mathrm{Si} / \mathrm{Ge}=55)$ : Although no experimental study reports an ITQ-13 silicogermanate with such a low Ge content, ITQ-13 models with 1 Ge/u.c. were constructed to investigate the site preference of $\mathrm{Ge}$ in the absence of any potential Ge-Ge interactions. Upon the removal of disorder, all symmetry elements with the exception of the A-centering are lost. Therefore, 28 of the $56 \mathrm{~T}$ sites in the unit cell become non-equivalent, and all of these were considered as Ge sites. Table S1.1 of the Supporting Information contains the relative energies $\Delta E$ with respect to the most favourable configuration (for which $\Delta E=0$ by definition) as well as the unit cell parameters. In the lowest-energy configuration, Ge is located at the T7 site to which F2 is bonded (labelled T7_1 in Table S1.1), forming a $\left[\mathrm{GeO}_{4} \mathrm{~F}\right]^{-}$trigonal bipyramid $\left(\left[4 \cdot 5^{6}\right]_{1 \mathrm{Ge}}\right.$, Figure $\left.3 \mathrm{e}\right)$. The F2-Ge distance of $1.90 \AA$ is considerably shorter than typical F-Ge bonds in $d 4 r$ units (discussed below), ${ }^{[27]}$ but exceeds the sums of covalent or ionic radii. ${ }^{[1,52]}$ The other T7 sites are at least $\sim 20 \mathrm{~kJ} \mathrm{~mol}^{-1}$ higher in energy (relative 
energies $\Delta E$ are always given in $\mathrm{kJ} \mathrm{mol}^{-1}$ per unit cell). In other low-energy configurations that are within 11 to $20 \mathrm{~kJ} \mathrm{~mol}^{-1}$ of the Ge@T7_1 configuration, Ge is located at the T2 or T5 sites, i.e., either of the T sites that are associated with the $d 4 r$ units. In these configurations, the F1 atom is always displaced from the cage centre towards the Ge atom, however, the individual F1-Ge distances vary considerably, from 2.12 to $2.57 \AA$ ( $d 4 r_{1 G e}$, Figure $3 \mathrm{a}$ ). The fact that all configurations are nevertheless close in energy indicates a very shallow potential energy surface for displacements of fluoride within the cage, as observed in a previous DFT study of AST-type silicogermanates. ${ }^{[27]}$ Configurations with Ge at any T site other than $\mathrm{T} 7, \mathrm{~T} 2$, or $\mathrm{T} 5$ are at least $39 \mathrm{~kJ} \mathrm{~mol}^{-1}$ higher in energy, agreeing perfectly with the experimental observation that only these three $\mathrm{T}$ sites are occupied by $\mathrm{Ge}$ at $\mathrm{Si} / \mathrm{Ge}$ ratios as low as $\sim 6 .{ }^{[4]} \mathrm{It}$ is interesting to note that some of the other sites $(\mathrm{T} 1, \mathrm{~T} 4, \mathrm{~T} 8, \mathrm{~T} 9)$ are high in energy despite being associated with 4MRs. In other words, while all preferred Ge locations are associated with 4MRs, not all T sites associated with 4MRs are preferred Ge locations. The strong energetic preference for these three sites is greatly reduced when considering a bare-framework model of ITQ-13 (Table S1.2), where the T6 and T9 sites are very close in energy to the T5 site, and even lower in energy than T2 and T7.

a) $d 4 r_{1 G e}$

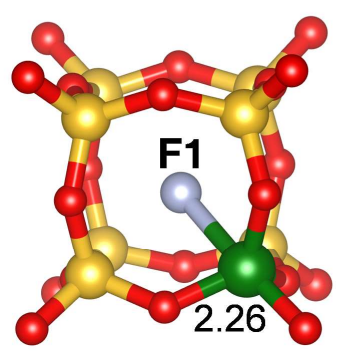

b) $d 4 r_{2 G e, p a i r}$

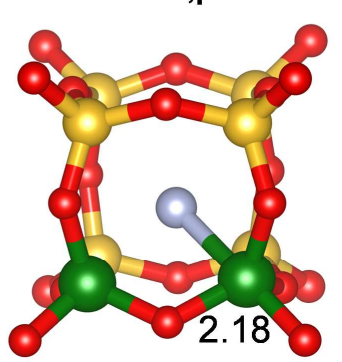

e) $\left[4 \cdot 5^{6}\right]_{1 \mathrm{Ge}}$

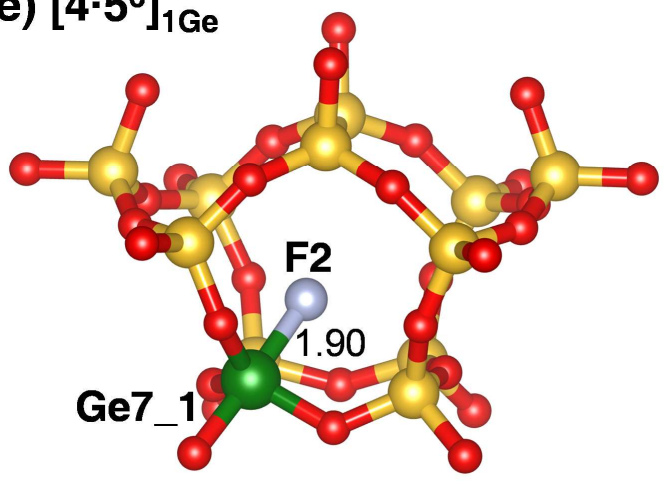

c) $d 4 r_{3 \mathrm{Ge}, 2 \mathrm{Ge}-\mathrm{O}-\mathrm{Ge}}$

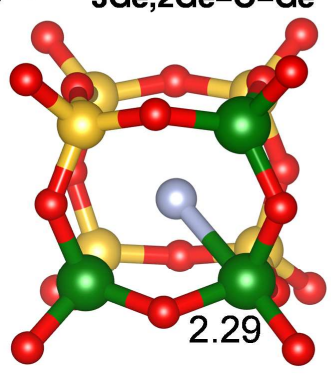

d) $d 4 r_{4 \mathrm{Ge}, 4 \mathrm{Ge}-\mathrm{O}-\mathrm{Ge}}$

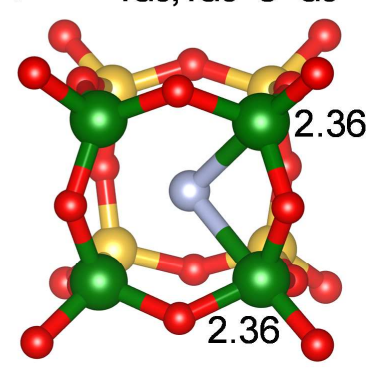

f) $\left[4 \cdot 5^{6}\right]_{2 G e, p a i r}$

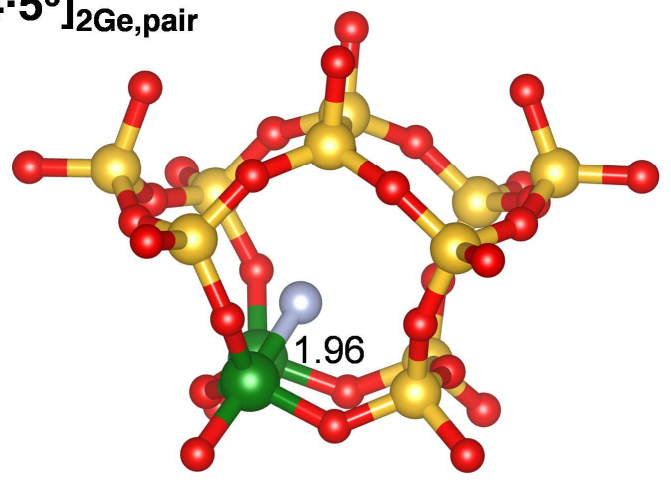

Figure 3: Representative examples of fluoride environments in DFT-optimised structures of Gecontaining ITQ-13 (green = germanium). F-Ge contacts shorter than $2.4 \AA$ are visualised as bonds. While representative $\mathrm{F}-\mathrm{Ge}$ distances are indicated (in $\AA$ ), these distances show a considerable variation among different structures containing identical building units in some instances. 
Due to the clear preference of Ge for the T7, T2, and T5 sites, the calculations for higher Ge contents considered only these three sites. For each Ge content, representative configurations were generated to sample different distributions. A sampling of all possible arrangements quickly becomes unfeasible due to the very large number of configurations: For $k \mathrm{Ge}$ atoms per unit cell, there are $24 ! /((24-k) ! k !)$ distinct arrangements, corresponding to $276 / 2,024 / 10,626 / 134,596 / 735,471$ configurations for $2 / 3 / 4 / 6 / 8$ Ge atoms per unit cell. Even after removing symmetry-equivalent configurations, a huge number remains: To illustrate this for the case of $6 \mathrm{Ge} / \mathrm{u}$.c., the number of nonequivalent combinations was calculated using the Supercell code ${ }^{[53]}$ for an ITQ-13 model without fluoride and OSDA (space group Amm2). This resulted in 17,029 distinct configurations, a number that is still much too large to be practically tractable with DFT calculations. Moreover, this is an underestimate of the total number of distinct configurations, as the inclusion of fluoride anions and OSDA molecules greatly reduces the number of symmetry elements, resulting in fewer equivalences.

The results of these calculations are compiled in Tables S2.1 to S6.2, and all optimised structures are supplied in PDB format at https://doi.org/10.6084/m9.figshare.17067752. In these tables and files, labels of the following format are used: ITQ-13_kGe_Y_ZZZ. Here, $k$ represents the number of Ge atoms per unit cell. $Y$ corresponds to a letter that is used to group configurations with similar Ge distributions together. $Y=A$ always corresponds to models where all Ge atoms occupy $\mathrm{T} 7$ sites, and $\mathrm{B}, \mathrm{C} \ldots$... represent configurations where an increasing number of $d 4 r$ sites are occupied. Separate letters are assigned to groups of configurations having a different distribution of Ge among the two $d 4 r$ cages. For example, in the ITQ-13_4Ge case, $G$ is used for a 2:2 distribution, $H$ for a 3:1 distribution, and I for a 4:0 distribution. Finally, $Z Z Z$ is an index number that is assigned to generate a unique label for each configuration. Where different configurations in one group contain different numbers of $\mathrm{Ge}-\mathrm{O}-\mathrm{Ge}$ linkages, the enumeration begins with those having the largest number of such linkages.

$2 \mathrm{Ge}$ atoms per unit cell $(\mathrm{Si} / \mathrm{Ge}=\mathbf{2 7})$ : A total of 18 configurations with $2 \mathrm{Ge}$ atoms per unit cell were considered (Tables S2.1 and S2.2). In the energetically most favourable model, T7 sites in both [4.5 $]$ cages are occupied by $\mathrm{Ge}$, in accordance with the results for $1 \mathrm{Ge} /$ u.c. Configurations in which two adjacent $\mathrm{T} 7$ sites in the same cage or one $\mathrm{T7}$ and one $d 4 r$ site (T2/T5) are occupied are about 10 to $15 \mathrm{~kJ} \mathrm{~mol}^{-1}$ less favourable. If the two Ge atoms are both located at $d 4 r$ sites, "distributed" configurations with two $d 4 r_{1 G e}$ cages are similar in energy to some configurations containing a single Ge-O-Ge linkage in one cage $\left(d 4 r_{2 G e, p a i r}\right.$, Figure $3 \mathbf{~ b}$ ), being about $25 \mathrm{~kJ} \mathrm{~mol}^{-1}$ above the lowest-energy scenario. The location of both Ge atoms at non-neighbouring $T$ sites belonging to the same $d 4 r$ cage sites is distinctly less favourable.

$3 \mathrm{Ge}$ atoms per unit cell (Si/Ge $=\mathbf{1 7 . 7 )}$ : The results for the 28 configurations containing $3 \mathrm{Ge} /$ u.c. are compiled in Tables S3.1 and S3.2. A distributed arrangement with two $\left[4 \cdot 5^{6}\right]_{1 \mathrm{Ge}}$ cages and one $d 4 r_{1 G e}$ 
cage emerges as the most favourable scenario, however, a model with one $\left[4 \cdot 5^{6}\right]_{2 G \text {, pair }}$ cage (Figure 3 f), where an adjacent T7 site instead of a $d 4 r$ site is occupied by the third Ge atom, is very close in energy $\left(\Delta E=3.8 \mathrm{~kJ} \mathrm{~mol}^{-1}\right)$. Configurations where $2 \mathrm{Ge}$ atoms are associated with $d 4 r$ cages are at least $10 \mathrm{~kJ} \mathrm{~mol}^{-1}$ higher in energy, and those in which all $3 \mathrm{Ge}$ atoms are located at $d 4 r$ sites are at least $28 \mathrm{~kJ} \mathrm{~mol}^{-1}$ less favourable. In the latter case, a $2+1$ distribution over the two cages is generally favoured over a localisation of all $\mathrm{Ge}$ atoms at corners of the same cage. Among the set of configurations in which all Ge atoms belong to the same $d 4 r$ cage, there is a clear tendency to maximise the number of Ge-O-Ge linkages, replicating the findings of previous work on AST-type systems. ${ }^{[27]}$

$4 \mathrm{Ge}$ atoms per unit cell (Si/Ge = 13): For a Ge content of $4 \mathrm{Ge} /$ u.c., 43 configurations were included in the calculations (Tables S4.1 and S4.2). As could be anticipated from the results for lower Ge contents, the most favourable configuration corresponds to a distribution of the Ge atoms over all four available cages, i.e., a configuration containing two $\left[4 \cdot 5^{6}\right]_{1 G e}$ cages and two $d 4 r_{1 G e}$ cages. A configuration where Ge atoms are exclusively located at T7 sites (two $\left[4 \cdot 5^{6}\right]_{2 \mathrm{Ge}, \text { pair }}$ cages) is, however, only slightly less favourable $\left(\Delta E=5.5 \mathrm{~kJ} \mathrm{~mol}^{-1}\right)$. Comparatively small $\Delta E$ values of less than $10 \mathrm{~kJ} \mathrm{~mol}^{-1}$ are also obtained if the Ge atoms are distributed over two $\left[4 \cdot 5^{6}\right]_{1 \mathrm{Ge}}$ cages and one $d 4 r_{2 \mathrm{Ge} \text {,pair }}$ cage. If at least one of the $\left[4 \cdot 5^{6}\right]$ cages has no Ge atom in the $4 \mathrm{MR}$, this will incur an energy penalty, with all configurations containing one all-silica $\left[4 \cdot 5^{6}\right]_{\mathrm{OGe}}$ cage being disfavoured by at least $20 \mathrm{~kJ} \mathrm{~mol}^{-1}$ compared to the lowest-energy case. When placing all $4 \mathrm{Ge}$ atoms at corners of the $d 4 r$ cages, configurations that have a 2:2 distribution are favoured over those with a 3:1 or 4:0 distribution. Within each group, a tendency to favour configurations having the largest possible number of Ge-O-Ge linkages is still present. However, the scatter in the $\Delta E$ values obtained for configurations having an identical number of such links is often considerable.

$6 \mathrm{Ge}$ atoms per unit cell $(\mathrm{Si} / \mathrm{Ge}=\mathbf{8 . 3})$ : A total number of 48 configurations with $6 \mathrm{Ge} /$ u.c. were compared (Tables S5.1 and 5.2). As this constitutes the largest number of distinct models for any Ge content, the results are presented in particular detail. The $\Delta E$ values of all models are visualised in Figure 4, grouped hierarchically according to 1 ) the overall distribution of Ge atoms between $\left[4 \cdot 5^{6}\right]$ and $d 4 r$ cages, 2) the distribution of the Ge@d4r atoms among the two cages, and 3) the total number of Ge-O-Ge links in the structure. The energetically most favourable configuration, indicated by an orange star, contains two $\left[4 \cdot 5^{6}\right]_{1 G e}$ cages and two $d 4 r_{2 G e, p a i r}$ cages. It is important to note that some other models having the same combination of building units are about $25 \mathrm{~kJ} \mathrm{~mol}^{-1}$ higher in energy (Figure 4, orange columns). Apparently, different ways to assemble the same types of cages may lead to rather different total energies, depending on their respective arrangement and orientation. This implies that the total energy is not solely determined by the local environments, but that there are 
other factors at play that cannot be captured using simple criteria like the number of Ge atoms in the constituent cages or the number of $\mathrm{Ge}-\mathrm{O}-\mathrm{Ge}$ linkages.

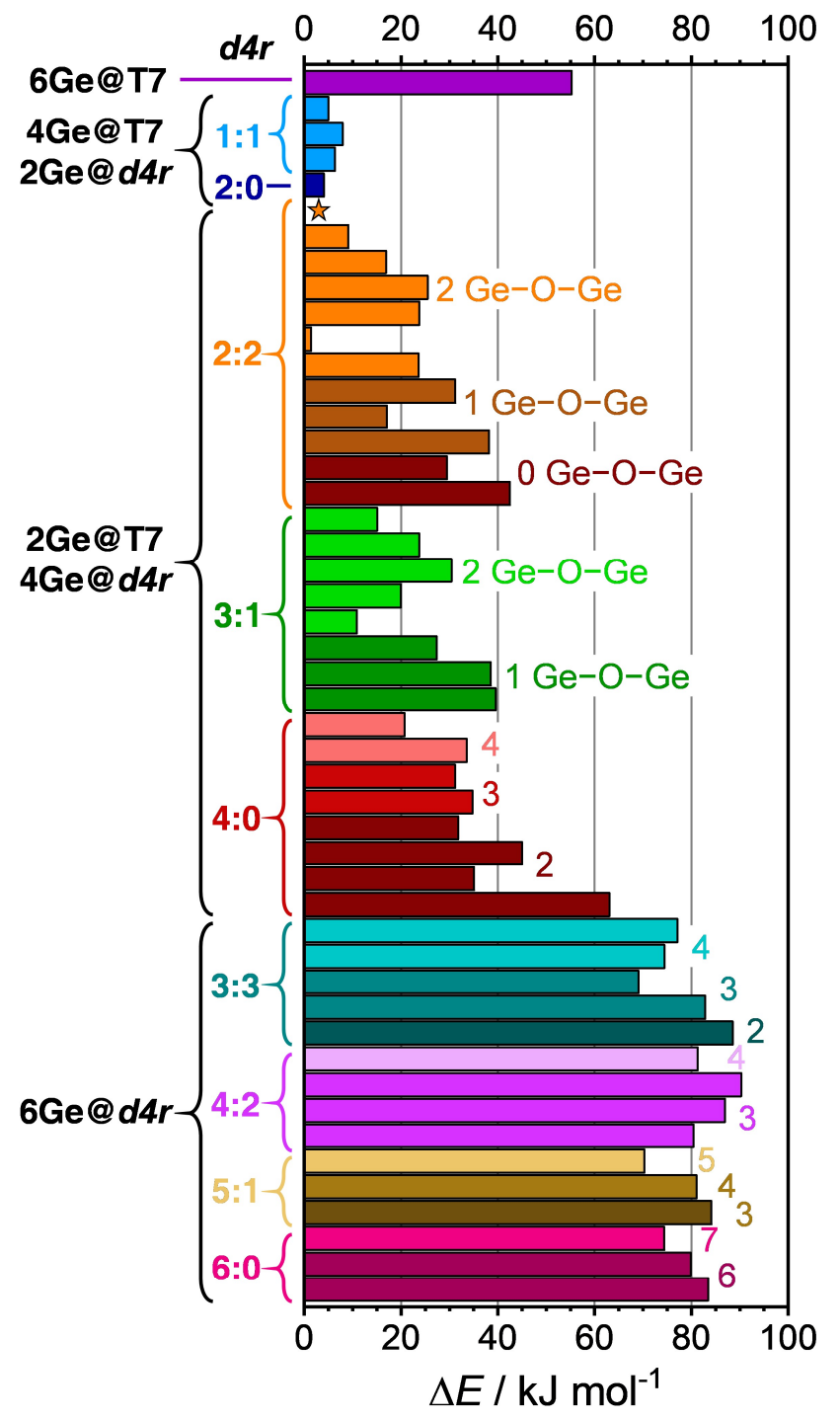

Figure 4: Relative stability of different Ge distributions in ITQ-13 with $6 \mathrm{Ge} / \mathrm{u} . \mathrm{c}$. , ordered according to the distribution among $\left[4 \cdot 5^{6}\right]$ and $d 4 r$ cages (black brackets), distribution among the two $d 4 r$ cages in the unit cell (coloured brackets), and number of Ge-O-Ge links (labels \& individual colours). The lowest-energy configuration is indicated by an orange star.

All models having two $\left[4 \cdot 5^{6}\right]_{2 \mathrm{Ge} \text {,pair }}$ cages are within 4 to $8 \mathrm{~kJ} \mathrm{~mol}^{-1}$ of the most favourable configuration, regardless of the Ge distribution among the two $d 4 r$ cages (Figure 4, blue columns). Therefore, it seems reasonable to expect that such cages are present in ITQ-13 samples with Si/Ge ratios below $\sim 10$. Conversely, the occupation of a third T7 site by Ge is energetically very unfavourable (purple column). Returning to the large number of configurations containing two Ge@T7 and four Ge@d4r atoms, the two aforementioned trends to a) distribute Ge evenly over the available cages (2:2 distribution 
favoured over 3:1 and 4:0) and to b) maximise the number of Ge-O-Ge linkages are still detectable. However, the two trends counteract each other, as the maximal number of possible Ge-O-Ge links increases with the number of Ge atoms at the corners of a single cage. Therefore, a 4:0 distribution with $4 \mathrm{Ge}-\mathrm{O}-\mathrm{Ge}$ linkages ( $1^{\text {st }}$ bright red column) has a smaller $\Delta E$ value smaller than both the least favourable 2:2 distribution with $2 \mathrm{Ge}-\mathrm{O}-\mathrm{Ge}$ linkages and either of the two 2:2 distributions with no Ge-O-Ge linkages that were included (brown columns). The same effect is visible for the configurations with six Ge@d4r atoms: While the $\Delta E$ values of all these models are large, amounting to at least $69 \mathrm{~kJ} \mathrm{~mol}^{-1}$, they are relatively close together in energy. The most favourable configurations with a 5:1/6:0 distribution, with 5/7 Ge-O-Ge links, lie only $1 / 5 \mathrm{~kJ} \mathrm{~mol}^{-1}$ above the lowest-energy 3:3 distribution (3 Ge-O-Ge links).

$8 \mathrm{Ge}$ atoms per unit cell $(\mathrm{Si} / \mathrm{Ge}=6)$ : The results for the 28 configurations with $8 \mathrm{Ge} / \mathrm{u}$.c. are compiled in Table S6.2. The lowest-energy configuration contains two $\left[4 \cdot 5^{6}\right]_{2 G e, p a i r}$ cages and two $d 4 r_{2 G e, p a i r}$ cages, but some models with an uneven distribution of the four Ge@d4r atoms among the two cages are reasonably close in energy, with $\Delta E$ in the range of 11 to $16 \mathrm{~kJ} \mathrm{~mol}^{-1}$. Configurations in which either more or less than two Ge atoms are associated with the $\left[4 \cdot 5^{6}\right]$ cages are distinctly less favourable (all $\Delta E$ values $>38 \mathrm{~kJ} \mathrm{~mol}^{-1}$ ), pointing to a strong tendency to favour $\left[4 \cdot 5^{6}\right]_{2 \mathrm{Ge} \text {,pair }}$ cages at relatively high Ge contents. For configurations with six or eight Ge@d4r atoms, the respective $\Delta E$ values show rather large variations. Not all of the individual differences can be explained as being due to the distribution of Ge among the two $d 4 r$ cages and/or the number of Ge-O-Ge links. A particularly interesting case is the ITQ-13_8Ge_K_003 configuration: It contains two $d 4 r_{4 G e, 4 G e-0-G e}$ cages, with the Ge-containing 4MRs lying perpendicular to the $a$ axis. Among those configurations having all $8 \mathrm{Ge}$ atoms at the corners of $d 4 r$ units, this model is the lowest in energy, being about $13 \mathrm{~kJ} \mathrm{~mol}^{-1}$ more favourable than the second-lowest energy case. The presence of $d 4 r_{4 G e, 4 G e-0-G e}$ cages has been linked to the complete disassembly of Ge-rich ITQ-13 samples ( $\mathrm{Si} / \mathrm{Ge}<3$ ) into layered solids upon acid treatment, as a removal of such an all-Ge 4MR would result in a disintegration of the $d 4 r$ units, disconnecting the layers. ${ }^{[13]}$ Based on the results of the present work, it can be expected that at least $12 \mathrm{Ge} /$ u.c. (4 at T7 sites, 8 at $d 4 r$ sites) would be required for a successful disassembly. The sample for which a complete disassembly was achieved had an even higher Ge content ( $\mathrm{Si} / \mathrm{Ge}=2.5$, corresponding to about 16 Ge/u.c.).

To summarise, the DFT study of ITQ-13 models containing between 1 and 8 Ge atoms per unit cell allows to draw the following conclusions:

- Ge atoms preferentially locate at the T sites forming the basal 4MR plane of the $\left[4 \cdot 5^{6}\right]$ cages ( $T 7$ site) and at the sites forming the $d 4 r$ cages ( $T 2+T 5$ site). As all other possible locations are much higher in energy, it is unlikely that they will be occupied by Ge at all. ITQ-13 samples with 
$\mathrm{Si} / \mathrm{Ge}$ ratios below 3 have been synthesised. ${ }^{[13]}$ Even such Ge-rich compositions could be achieved by occupying exclusively the $T 7$ and $d 4 r$ sites, with an occupation of $1 / 2$ of the $T 7$ sites and all $d 4 r$ sites ( $=4+16=20 \mathrm{Ge} /$ u.c.) corresponding to an Si/Ge ratio of 1.8.

- Agreement with the experimentally observed preference of Ge for the T2, T5, and T7 sites ${ }^{[4]}$ is achieved only with models containing fluoride anions and OSDA cations. This observation confirms the previous notion that calculations for bare-framework models are of limited validity to predict the most likely Ge location(s), as they ignore the important role of fluoride anions and OSDA cations in stabilising the preferred Ge sites. ${ }^{[27]}$ Since only hexamethonium cations were considered, it cannot be inferred whether a change of the OSDA would lead to a variation in the site preference, or whether it is governed solely by the local environment and interactions with the fluoride anions.

- With regard to the $\left[4 \cdot 5^{6}\right]$ cages, an occupation of one T7 site by Ge is strongly favoured. It can thus be expected that the amount of all-silica $\left[4 \cdot 5^{6}\right]_{\text {oge }}$ cages will be very limited if there are more than $\sim 4$ Ge atoms per unit cell (Si/Ge $>13$ ). Indeed, the corresponding ${ }^{19} \mathrm{~F}-\mathrm{NMR}$ resonance at $\delta=-66 \mathrm{ppm}$, which is strong in ITQ-13_SiO $\mathrm{Si}_{2}$ and in samples with an $\mathrm{Si} / \mathrm{Ge}$ ratio above 20 , is much weaker in more Ge-rich samples (Table $1^{[4,11]}$ ). The occupation one T7 site by $\mathrm{Ge}$, predicted by the calculations even for very low Ge contents, is confirmed experimentally through the appearance of a new resonance at $\delta=-55 \mathrm{ppm}$, which is absent in ITQ-13_SiO ${ }_{2}$. This peak is visible even in the most Ge-rich sample investigated by Vidal-Moya et al. ( $\mathrm{Si} / \mathrm{Ge}=$ 25 , equivalent to about $2 \mathrm{Ge} /$ u.c.). ${ }^{[4]}$

- At higher Ge contents, $\left[4 \cdot 5^{6}\right]_{2 G e, p a i r}$ cages are energetically favourable. The possible presence of this type of cage has not yet been discussed in the experimental literature.

- With regard to the $d 4 r$ cages, a distribution of Ge among the available cages is energetically favoured over a coexistence of all-silica cages and cages having Ge atoms at several corners, even at low Ge contents. This prediction is in line with the rapid intensity decrease of the ${ }^{19} \mathrm{~F}$ NMR resonance at $\delta=-38 \mathrm{ppm}$, which stems from fluoride incorporated in $d 4 r_{0 G e}$ cages, at $\mathrm{Si} / \mathrm{Ge}$ ratios below 20 (more than $\sim 3 \mathrm{Ge} /$ u.c.). At higher Ge contents, different distributions of Ge among the $d 4 r$ cages, as well as different arrangements within a given cage, are relatively close in energy, pointing to a coexistence of a multitude of local environments. As a consequence, the ${ }^{19} \mathrm{~F}-\mathrm{NMR}$ resonance at $\delta=-20 \mathrm{ppm}$, indicative of $d 4 \mathrm{r}$ cages having isolated Ge atoms at one or several corners, remains detectable up to an Si/Ge ratio of 4.5 ( 10 Ge/u.c.), even though there are, on average, at least 3 Ge atoms per $d 4 r$ cage at this Ge content. For a given number of Ge atoms per cage, arrangements having a larger number of Ge-O-Ge links tend to be favoured. 
Some significant approximations had to be made: First, the DFT optimisations deliver only the total energy at $0 \mathrm{~K}$, ignoring thermal effects. Although this approximation may affect the energetic ordering of configurations that are relatively close in energy, it is unlikely that the qualitative trends identified above would be changed. Second, the calculations can only predict the relative stabilities of the periodic zeolite structures. In zeolite synthesis, the actual distribution of $\mathrm{Ge}$ in the final product will be determined during the formation of small building units (such as individual $d 4 r$ cages), which then assemble to form the extended structure. The periodic DFT calculations performed here capture neither the thermodynamic stability of such discrete building units nor their formation kinetics. Despite these inevitable limitations, the good agreement of the calculation results with the available experimental data gives reason to be confident that the chosen approach is suitable to predict probable low-energy configurations.

\section{Prediction of ${ }^{19} \mathrm{~F}-\mathrm{NMR}$ chemical shifts}

As mentioned in the previous section, the occurrence of $\left[4 \cdot 5^{6}\right]_{2 G e, p a i r}$ cages with two neighbouring Ge atoms in the basal 4MR plane has not yet been proposed in experimental studies. In particular, there is no unassigned ${ }^{19} \mathrm{~F}$ chemical shift that could correspond to fluoride anions in these cages. However, it is possible that the resonance stemming from these cages overlaps with another peak. This might obscure the presence of an additional building unit, especially if the resonance is rather weak. To test this hypothesis, calculations of the ${ }^{19} \mathrm{~F}-\mathrm{NMR}$ chemical shifts were carried out for a total of six building units. For five of these cages, $d 4 r_{0 G e}, d 4 r_{1 G e}, d 4 r_{2 G e, p a i r}\left[4 \cdot 5^{6}\right]_{0 G e}$, and $\left[4 \cdot 5^{6}\right]_{1 G e}$, the (approximate) shifts are known, whereas the sixth building unit is the $\left[4 \cdot 5^{6}\right]_{2 G \text { e,pair }}$ cage. Non-periodic cluster models were extracted from the DFT-optimised structures, and the isotropic chemical shifts were computed using the PBEO and B3PW91 functionals with cc-pVTZ basis sets. The results are compiled in Table 2.

Table 2: ${ }^{19} \mathrm{~F}$ chemical shifts obtained from calculations on cluster models employing the PBEO and B3PW91 hybrid exchange-correlation functionals. Differences with respect to the experimental values are given in brackets.

\begin{tabular}{lccc}
\hline \hline & $\delta_{\text {exp }} / \mathrm{ppm}$ & $\delta_{\mathrm{DFT}}(\mathrm{PBEO}) / \mathrm{ppm}$ & $\delta_{\mathrm{DFT}}(\mathrm{B} 3 \mathrm{PW} 91) / \mathrm{ppm}$ \\
\hline$d 4 r_{0 G \mathrm{Ge}}$ & -38 & $-44.1(-6.1)$ & $-46.7(-8.7)$ \\
$d 4 r_{1 \mathrm{Ge}}$ & -20 & $-20.0(0.0)$ & $-21.2(-1.2)$ \\
$d 4 r_{2 \mathrm{Ge}, \mathrm{pair}}$ & -8 & $-3.5(4.5)$ & $-4.7(3.3)$ \\
{$\left[4 \cdot 5^{6}\right]_{0 \mathrm{Ge}}$} & -66 & $-62.2(3.8)$ & $-67.3(-1.3)$ \\
{$\left[4 \cdot 5^{6}\right]_{1 \mathrm{Ge}}$} & -55 & $-48.6(6.4)$ & $-50.4(4.6)$ \\
{$\left[4 \cdot 5^{6}\right]_{2 \mathrm{Ge}, \mathrm{pair}}$} &.$/$ & -15.7 & -16.6 \\
\hline \hline
\end{tabular}


Comparing the computed ${ }^{19} \mathrm{~F}-\mathrm{NMR}$ chemical shifts to the experimental reference values, the mean of absolute errors is similar for both functionals, amounting to $4.2 \mathrm{ppm}$ for PBE0 and $3.8 \mathrm{ppm}$ for B3PW91. In view of the limited number of experimental data points, and the lack of any specific optimisation of the computational approach (e.g., in terms of the combination of exchange-correlation functional and basis set), such an agreement appears satisfactory, and sufficiently good to draw semi-quantitative conclusions on the resonance stemming from fluoride anions in $\left[4 \cdot 5^{6}\right]_{2 G e, p a i r}$ cages. As shown in Table 2, both functionals give very similar shifts in the range of $\delta=-16 \mathrm{ppm}$ for these fluoride anions. In other words, the associated resonance should fall between the two peaks at $\delta=-8 \mathrm{ppm}$ ( $d 4 \mathrm{r}$ cages with at least one Ge-O-Ge link) and $\delta=-20 \mathrm{ppm}$ ( $d 4 \mathrm{r}$ cages with isolated Ge atoms). Both peaks are rather broad, and in some Ge-rich samples, the intensity between them does not fall to zero. ${ }^{[11]}$ Also, the broad peak at $\delta=-8 \mathrm{ppm}$ does not appear to be fully symmetric, with a shoulder in the range of $\delta=$ 11 to -14 ppm (best visible in Figure $3 \mathrm{c}$ ) and $3 \mathrm{~d}$ ) of ref. ${ }^{[11]}$ ). Given the uncertainty of the DFT-based prediction of the chemical shift, which is on the order of $5 \mathrm{ppm}$, it is possible that the resonance stemming from fluoride-containing $\left[4 \cdot 5^{6}\right]_{2 G e, p a i r}$ cages was so far not observed in experimental studies due to its overlap with the peaks at $\delta=-8$ or $-20 \mathrm{ppm}$.

\section{Dynamics of fluoride anions}

In order to investigate the dynamics of the fluoride anions, AIMD simulations were performed for temperatures of $298 \mathrm{~K}$ and $408 \mathrm{~K}$ for a total of 10 different ITQ-13 models with 0 to $8 \mathrm{Ge}$ atoms per unit cell. The latter temperature was chosen because it is typical for the synthesis of ITQ-13. ${ }^{[2]}$ Table S7.1 indicates for which ITQ-13 models these simulations were carried out, and the AIMD trajectories are available at https://doi.org/10.6084/m9.figshare.17067752 (production stage, in PDB format). The RMSDs were computed for the fluoride anions incorporated in distinct building units. The reported RMSD(F) values, compiled in Table 3 for $\left[4 \cdot 5^{6}\right]$ cages and in Table $\mathbf{4}$ for $d 4 r$ cages, are averages over all trajectories in which such building units are present (individual values are collected in Tables $\mathbf{S 7 . 2}$ and 7.3). The number $N$ of individual RMSD values included in the calculation of the averages is also given. $N$ ranges from 3, corresponding to building units appearing only once in one ITQ-13 model, to 30 . A visualisation of the individual RSMD(F) datapoints is included in the Supporting Information (Figures S6.1 and S6.2). For each environment, representative fluoride trajectories were plotted into the average structure of the surrounding cage to highlight key features of the dynamic behaviour. 
Fluoride in $\left[4 \cdot 5^{6}\right]$ cages, $\boldsymbol{T}=\mathbf{2 9 8} \mathrm{K}$ : The RMSD of fluoride anions incorporated in all-silica $\left[4 \cdot 5^{6}\right]_{0 G e}$ cages amounts to $0.22 \AA$ at $298 \mathrm{~K}$. A very similar value was previously obtained for a model of STF-type Mu26, which contains the same type of cage. ${ }^{[48]}$ The plot of all individual values (Figure S6.1) confirms the absence of any increased RMSDs that would be indicative for a dynamic exchange of fluoride between different Si atoms. ${ }^{[16,48,54,55]}$ The fluoride anions only undergo oscillations about their equilibrium positions at the apex of the $\left[\mathrm{SiO}_{4} \mathrm{~F}\right]^{-}$trigonal bipyramids, as illustrated in Figure $\mathbf{5}$ a. The average RMSD(F) obtained for fluoride anions in $\left[4 \cdot 5^{6}\right]_{1 \mathrm{Ge}}$ cages is only slightly larger than for the all-silica case, indicating that $\mathrm{F}-\mathrm{Ge}$ bonds are somewhat more flexible than $\mathrm{F}-\mathrm{Si}$ bonds. For fluoride anions incorporated in $\left[4 \cdot 5^{6}\right]_{2 G e, p a i r}$ cages, the RMSD(F) is significantly larger, amounting to $0.27 \AA$. . An analysis of individual trajectories shows that this is due to a movement of fluoride between the Ge atoms, with similarly short contacts to both atoms occurring over the course of the trajectory. This behaviour is visualised in Figure 5 c. As a consequence of these oscillations, the average position of fluoride computed over an AIMD trajectory lies approximately at equal distance from both Ge atoms $(d(\mathrm{~F}-\mathrm{Ge})$ $\approx 2.2 \AA$ ), whereas one F-Ge contact is significantly shorter than the other in the DFT-optimised structures (in the representative cage shown in Figure $\mathbf{3} \mathbf{f}$, the F-Ge bond is $1.96 \AA$ long, while the distance to the other Ge atom amounts to $2.45 \AA$ ) $)$.

Table 3: RMSDs of fluoride anions located in $\left[4 \cdot 5^{6}\right]$ cages. All values were calculated as averages over the RMSDs computed for individual F positions in individual AIMD trajectories. The number of RMSD values $N$ is given in brackets. For $408 \mathrm{~K}$, both the overall RMSD(F) values and RMSD(F) computed considering only those fluoride anions that do not undergo dynamic events ("noDE") are reported. For comparison, the $298 \mathrm{~K}$ value from previous work on the STF-type all-silica zeolite Mu-26 is included. ${ }^{[48]}$

\begin{tabular}{|c|c|c|c|c|}
\hline & Zeolite & "RMSD(F)all, $298 \mathrm{~K} / \AA ̊$ & "RMSD(F) $)_{\text {all, }} 408 \mathrm{~K} / \AA$ & "RMSD(F) noDE, $408 \mathrm{~K} / \AA$ \\
\hline \multirow{4}{*}[4\cdot5^{6}]{$_{o G e}$} & \multirow{2}{*}{ ITQ-13 } & $0.224 \pm 0.011$ & $0.362 \pm 0.139$ & $0.265 \pm 0.015$ \\
\hline & & $(N=12)$ & $(N=12)$ & $(N=8)$ \\
\hline & STF, & $0.214 \pm 0.014$ & I & \\
\hline & F@Si10[48] & $(N=16)$ & $\%$ & . \\
\hline \multirow{2}{*}[4\cdot5^{6}]{$_{1 \mathrm{Ge}}$} & \multirow{2}{*}{ ITQ-13 } & $0.234 \pm 0.005$ & $0.290 \pm 0.030$ & $0.279 \pm 0.012$ \\
\hline & & $(N=18)$ & $(N=18)$ & $(N=15)$ \\
\hline \multirow{2}{*}[4\cdot5^{6}]{$_{2 G e, p a i r}$} & \multirow{2}{*}{ ITQ-13 } & $0.273 \pm 0.017$ & $0.317 \pm 0.015$ & \multirow{2}{*}{$=\operatorname{RMSD}(F)_{\text {all }}$} \\
\hline & & $(N=30)$ & $(N=30)$ & \\
\hline
\end{tabular}




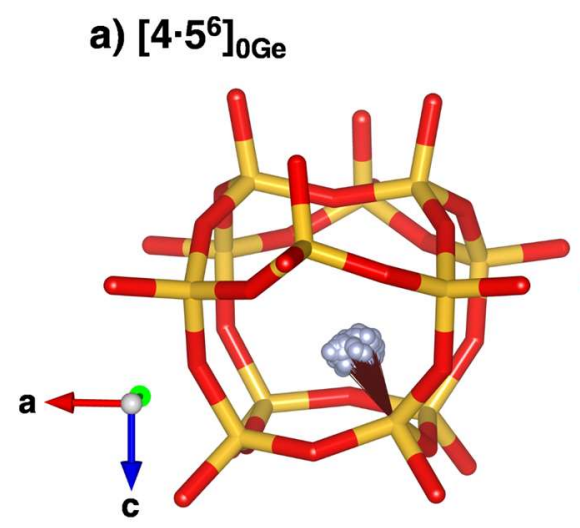

b) $\left[4 \cdot 5^{6}\right]_{1 \mathrm{Ge}}$

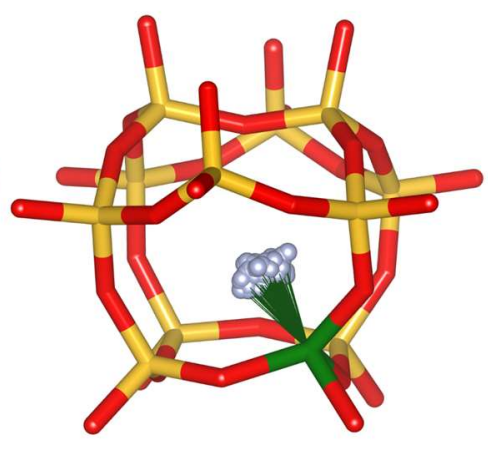

c) $\left[4 \cdot 5^{6}\right]_{2 G e, p a i r}$

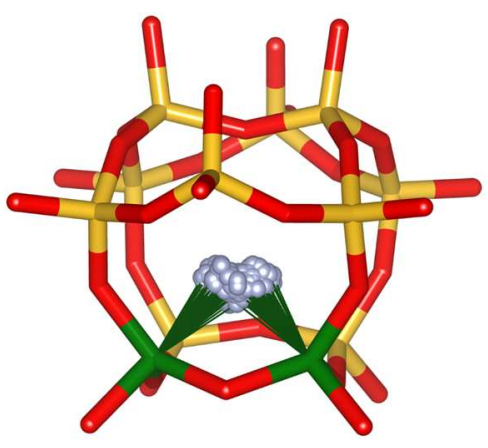

Figure 5: Representative trajectories of fluoride anions in $\left[4 \cdot 5^{6}\right]$ cages computed for $T=298 \mathrm{~K}$. In each case, the coordinates of the atoms at the corner of the cage are taken from the AIMD average structures. F-Si contacts below $1.8 \AA$ And F-Ge contacts below $2.0 \AA$ are indicated by thin lines.

Fluoride in [4.56] cages, $\boldsymbol{T}=408 \mathrm{~K}$ : Typical RMSD(F) values computed for fluoride in all-silica $\left[4 \cdot 5^{6}\right]_{0 G e}$ cages fall in the range of 0.25 to $0.30 \AA$ (Figure S6.1). The growth with respect to the $298 \mathrm{~K}$ values is attributed to the increased thermal motion at $408 \mathrm{~K}$ (for an harmonic oscillator, an increase of $(408 / 298)^{\wedge} 0.5 \approx 1.17$ would be expected ${ }^{[56]}$ ). It is, however, noteworthy that the RMSDs of some individual fluoride anions are significantly larger, typically exceeding $0.5 \AA$, and resulting in an average $\operatorname{RMSD}(\mathrm{F})_{\text {all }}$ of $0.36 \AA$ (Table 3). As established in previous work, such a drastic enhancement of the RMSD can be explained with dynamic "events" that correspond to a movement of the fluoride anion between different Si sites. ${ }^{[48,55]}$ Solid-state NMR measurements provide evidence for the roomtemperature dynamic disorder of the fluoride anions in some all-silica zeolites like Silicalite-1 and ITQ4. ${ }^{[16,54]}$ Although such measurements have not yet been reported for elevated temperatures, a previous AIMD study showed that the increased thermal motion may lead to the occurrence of dynamic events in zeolites that do not exhibit dynamic behaviour at room temperature, one example being STF-type Mu-26. ${ }^{[48]}$ It is hence not surprising that the same kind of behaviour is found in $\left[4 \cdot 5^{6}\right]_{0 G e}$ cages of ITQ-13. An evaluation of the atomic coordinates shows that ca. $1 \AA$ "jumps" may occur along the $a$ and $b$ directions. In one case, a single fluoride anion visits three $\mathrm{Si}$ atoms during the $15 \mathrm{ps}$ trajectory (Figure 6 a). If fluoride anions undergoing dynamic events are excluded from the calculation

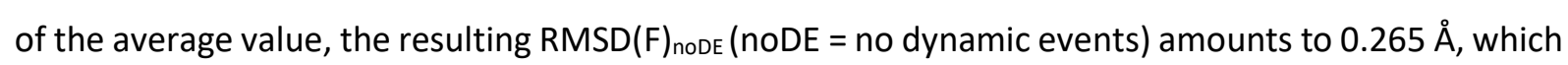
is $18 \%$ larger than the $298 \mathrm{~K}$ value.

Concerning fluoride anions incorporated in $\left[4 \cdot 5^{6}\right]_{1 G e}$ cages, some RMSD values fall outside the typical 0.26 to $0.30 \AA$ range. However, the individual increases are much less pronounced than for $\left[4 \cdot 5^{6}\right]_{\text {oGe }}$, and excluding these cases from the average RMSD(F) calculation results only in a modest change (Table 3). An evaluation of the individual trajectories of these fluoride anions reveals that such increases are 
due to short-lived jumps towards one of the surrounding Si atoms. Unlike observed above for the allsilica cages, the fluoride anions quickly return to the Ge atom, typically after a picosecond or less, and do not remain bonded to a Si atom for longer time intervals. A representative example of this behaviour is shown in Figure 6 b. Finally, the RMSDs of fluoride anions in $\left[4 \cdot 5^{6}\right]_{2 G e, p a i r}$ cages computed from the $408 \mathrm{~K}$ trajectories fall between 0.29 and $0.34 \AA$, and no increase of individual values beyond this range is observed. As illustrated in Figure $6 \mathrm{c}$, the fluoride anions rapidly move back and forth between the two Ge atoms, with no extended residence times at either site.
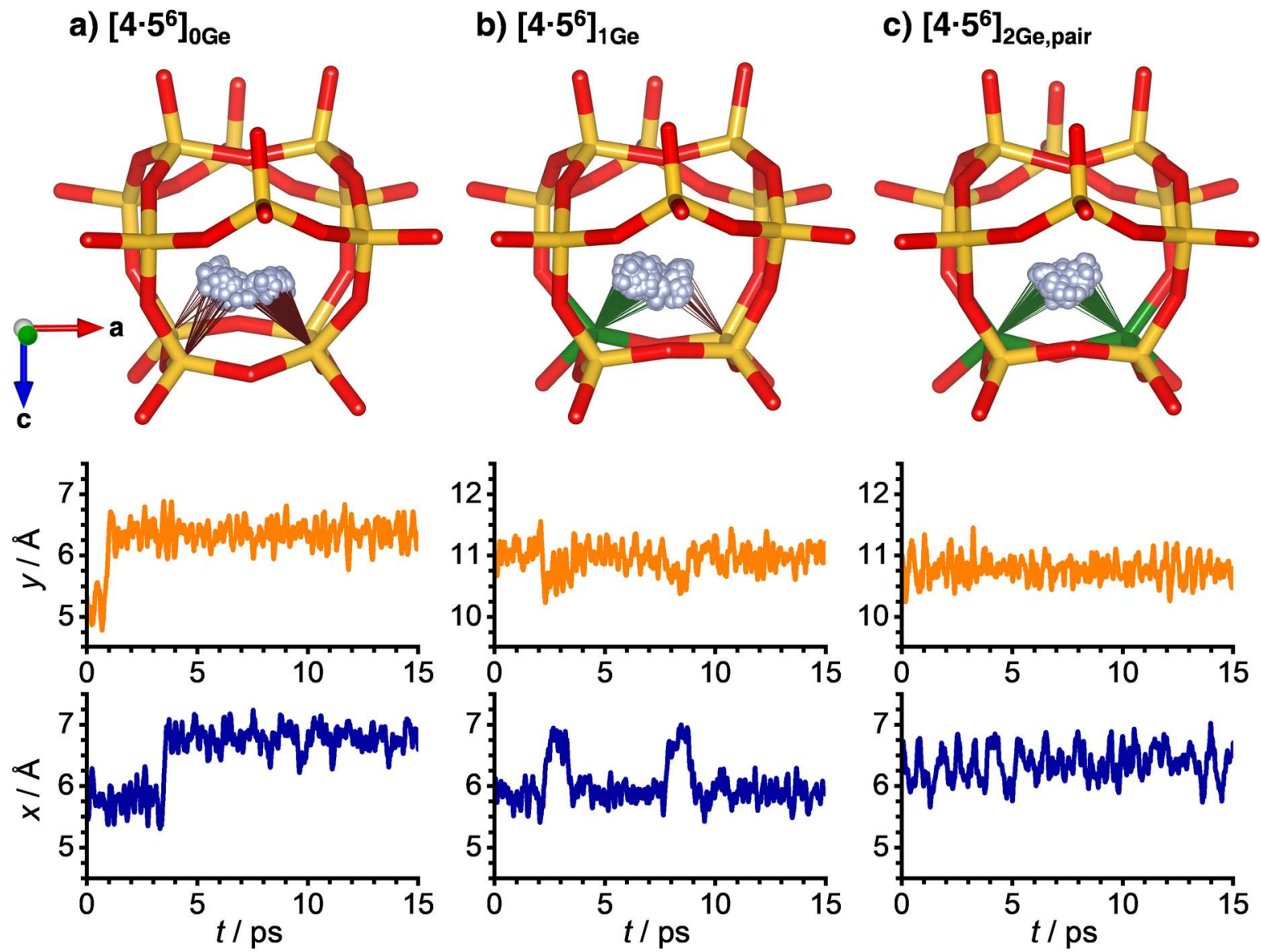

Figure 6: Top: Representative trajectories of fluoride anions in $\left[4 \cdot 5^{6}\right]$ cages computed for $T=408 \mathrm{~K}$. $\mathrm{F}-\mathrm{Si}$ contacts and F-Ge contacts below $2.0 \AA$ are indicated by thin lines. Bottom: Time evolution of the $x$ and $y$ coordinates of the fluoride anions.

Fluoride in $d 4 r$ cages, $T=298 \mathrm{~K}$ : The RMSD values computed for fluoride anions in different types of $d 4 r$ cages are compiled in Table 4 . As a fairly comprehensive study of the dynamics of fluoridecontaining $d 4 r$ cages has been reported previously for AST-type silicogermanates, ${ }^{[27]}$ encompassing a larger range of compositions up to $d 4 r_{8 G e}$ cages, the results for the relevant types of cages are also 
included. Due to the absence of localised F-Si bonds, fluoride anions in all-silica $d 4 r_{0 G e}$ cages possess a considerable freedom of motion, reflected by a fairly large RMSD(F) of $0.29 \AA$. . The formation of F-Ge bonds in $d 4 r$ cages containing 1 or $2 \mathrm{Ge}$ atoms results in drastically reduced RMSD(F) values. Because the fluoride anions in $d 4 r_{2 G e \text {,pair }}$ cages do not approach the Ge atoms as closely as in $\left[4 \cdot 5^{6}\right]_{2 G e, p a i r}$ cages, they remain confined to a narrower region between the two Ge atoms, and the corresponding RMSD is considerably smaller (compare Figure $\mathbf{6} \mathrm{c}$ and Figure $\mathbf{7 c} \mathrm{c}$ ). When more than two Ge atoms occupy corners of the $d 4 r$ cage, the RMSD(F) rises again because fluoride anions form short contacts to different Ge atoms over the course of the AIMD simulations. This trend was even clearer in the previous work on AST-type systems, especially when moving towards more Ge-rich $d 4 r$ cages (the RMSD(F) for a $d 4 r_{8 G e}$ cage amounts to $0.36 \AA$ ). ${ }^{[27]}$ Altogether, the quantitative deviations in the RMSDs between this and the previous study do not exceed $0.02 \AA$, which is plausible given the primary influence of the local environment on the freedom of motion.

Table 4: RMSDs of fluoride anions located in $d 4 r$ cages. Values from previous work on AST-type silicogermanates are included for comparison (298 K only). ${ }^{[27]}$

\begin{tabular}{|c|c|c|c|c|}
\hline & Zeolite & RMSD(F)all, $298 \mathrm{~K} / \AA$ & RMSD(F) all, $408 \mathrm{~K} / \AA$ & 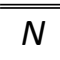 \\
\hline \multirow[t]{2}{*}{$d 4 r_{0 G e}$} & ITQ-13 & $0.285 \pm 0.011$ & $0.329 \pm 0.014$ & 21 \\
\hline & $\mathrm{AST}^{[27]}$ & 0.275 & $\%$ & 24 \\
\hline \multirow[t]{2}{*}{$d 4 r_{1 \mathrm{Ge}}$} & ITQ-13 & $0.217 \pm 0.019$ & $0.266 \pm 0.020$ & 21 \\
\hline & $\mathrm{AST}^{[27]}$ & 0.218 &. & 24 \\
\hline \multirow[t]{2}{*}{$d 4 r_{2 G e, p a i r}$} & ITQ-13 & $0.217 \pm 0.012$ & $0.266 \pm 0.012$ & 12 \\
\hline & $\mathrm{AST}^{[27]}$ & 0.232 &. & 24 \\
\hline \multirow[t]{2}{*}{$d 4 r_{3 G e, 2 G e-O-G e}$} & ITQ-13 & $0.226 \pm 0.009$ & $0.265 \pm 0.004$ & 3 \\
\hline & $\mathrm{AST}^{[27]}$ & 0.246 &. & 24 \\
\hline \multirow[t]{2}{*}{$d 4 r_{4 G e, 4 G e-O-G e}$} & ITQ-13 & $0.253 \pm 0.009$ & $0.309 \pm 0.007$ & 3 \\
\hline & $\mathrm{AST}^{[27]}$ & 0.264 &. & 24 \\
\hline
\end{tabular}

Fluoride in $\mathbf{d 4 r}$ cages, $\boldsymbol{T}=\mathbf{4 0 8} \mathrm{K}$ : The RMSD(F) values obtained for a temperature of $408 \mathrm{~K}$ are 15 to $22 \%$ larger than those computed for $298 \mathrm{~K}$, as expected. By and large, the same trends are found as for $298 \mathrm{~K}$. When comparing the results for $d 4 r_{2 \mathrm{Ge}, \text { pair }}$ to $d 4 r_{3 \mathrm{Ge}, 2 \mathrm{Ge}-\mathrm{O}-\mathrm{Ge}}$ cages, the slight increase of the RMSD observed for $298 \mathrm{~K}$ does not occur for $408 \mathrm{~K}$. However, this may be an artefact arising from the very limited sampling of the latter type of environment. 


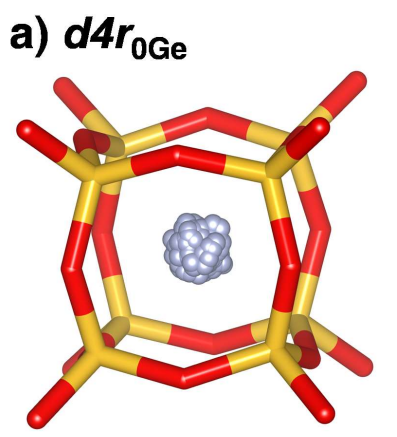

b) $d 4 r_{1 G e}$

c) $d 4 r_{2 G e, p a i r}$
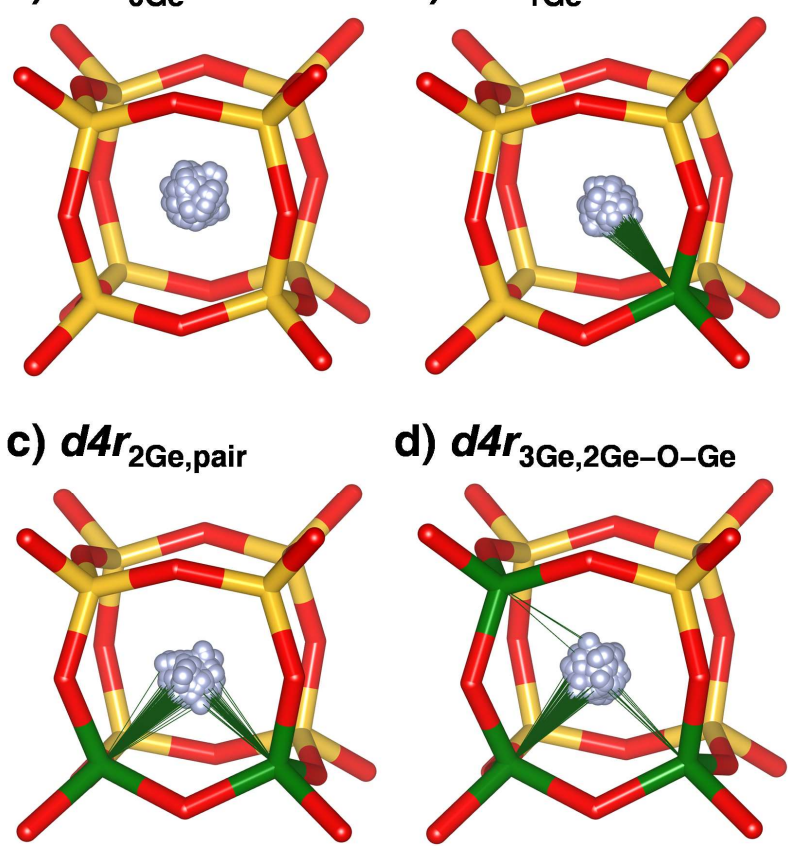

d) $d 4 r_{3 G e, 2 G e-0-G e}$

e) $d 4 r_{4 G e, 4 G e-0-G e}$

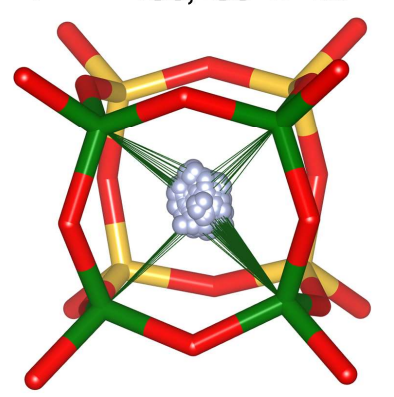

Figure 7: Representative trajectories of fluoride anions in $d 4 r$ cages computed for $T=298 \mathrm{~K}$. F-Ge contacts below $2.2 \AA$ are indicated by thin lines.

\section{Partial defluorination of ITQ-13}

Liu et al. subjected ITQ-13_SiO ${ }_{2}$ to an alkaline treatment. ${ }^{[10]}$ After 24 hours at $\mathrm{pH}=13.5$ and elevated temperature (448 K), the disappearance of the ${ }^{19} \mathrm{~F}-\mathrm{NMR}$ resonance at $\delta=-66 \mathrm{ppm}$ showed that the fluoride anions were completely removed from the $\left[4 \cdot 5^{6}\right]$ cages, whereas those in the $d 4 r$ cages remained unaffected. In a subsequent work on Ge-containing ITQ-13 samples, the same group demonstrated that the alkaline treatment is also effective in removing fluoride from $\left[4 \cdot 5^{6}\right]_{1 \mathrm{Ge}}$ cages (disappearance of the resonance at $\delta=-55 \mathrm{ppm}$ ), whereas only partial, if any, removal from $d 4 r$ cages took place for samples with low and intermediate Ge contents (Si/Ge $=21$ and 9). ${ }^{[11]}$ Only for the most Ge-rich samples, with Si/Ge ratios of 4.5 and 3.2, a near-complete removal from the $d 4 r$ cages occurred. These observations could be explained using different arguments. On the one hand, the 
different defluorination behaviour may be caused by differences in the thermodynamic stability of the fluoride environments. On the other hand, it could be related primarily to kinetic effects, such as a more facile release of fluoride from $\left[4 \cdot 5^{6}\right]$ cages through the larger $5 \mathrm{MR}$ windows. While the latter point would require a different computational approach, the relative stability of different environments can be assessed using DFT optimisations of partially defluorinated models.

Three different possibilities to generate partially defluorinated models were compared for ITQ13_ $\mathrm{SiO}_{2}$, in each case generating two models in which either of the two types of fluoride anions was removed. In the first approach, the remainder of the structure was left unchanged. Due to the uncompensated positive charge of the OSDA cations, a charge of $+2 /$ u.c. was assigned in the DFT calculations. In the second approach, 50\% of the OSDA nitrogen atoms were replaced by carbon to achieve neutrality. In the third approach, one of the two hexamethonium cations was removed, again resulting in a charge-neutral model. For each approach, the two models were optimised separately, with or without an optimisation of the unit cell parameters. The difference in stability of the two models was then expressed as $\Delta E\left(\mathrm{~F} @ d 4 r-\mathrm{F} @\left[4 \cdot 5^{6}\right]\right)$, where the label in brackets designates the type of fluoride anions still present in the structure. In other words, a negative value corresponds to a higher stability of the model containing fluoride only in the $d 4 r$ cages. The full results for all approaches tested on ITQ-13_SiO ${ }_{2}$ are compiled in Table S8.1. Despite the different approximations made, the values of $\Delta E\left(\mathrm{~F} @ d 4 r-\mathrm{F} @\left[4 \cdot 5^{6}\right]\right)$ are fairly similar, ranging from -47 to $-59 \mathrm{~kJ} \mathrm{~mol}^{-1}$ per fluoride anion. Regardless of the chosen approach, the removal from $\left[4^{\cdot} 5^{6}\right]$ cages is significantly more favourable than the removal from $d 4 r$ cages, in qualitative agreement with the experimental observations. This result indicates that the high thermodynamic stability of fluoride-containing $d 4 r$ cages does indeed contribute to the observed selective removal from $\left[4 \cdot 5^{6}\right]$ cages upon alkaline treatment.

Since the main aim of the following calculations was a qualitative prediction of the relative stability, calculations on partially defluorinated models of Ge-containing ITQ-13 employed only the third approach (removal of one OSDA per u.c.). While the results from the calculations with and without an optimisation of the cell parameters are compiled in Table $\mathbf{5 8 . 2}$, the present discussion focusses on calculations using a fixed cell, for which the resulting $\Delta E\left(\mathrm{~F} @ d 4 r-\mathrm{F} @\left[4 \cdot 5^{6}\right]\right)$ values are given in Table 5. Progressive incorporation of Ge atoms at the corners of the $\left[4 \cdot 5^{6}\right]$ cages while retaining all-silica $d 4 r_{0 G e}$ cages reduces the energy difference between the partially defluorinated models, with $\Delta E(\mathrm{~F} @ d 4 r$ F@[4.5 $]$ ) having only a slightly negative value for the $\left[4 \cdot 5^{6}\right]_{1 G e}$ case. This does not agree with the experimental observation of a selective defluorination of $\left[4 \cdot 5^{6}\right]_{1 G e}$ cages evidenced by the disappearance of the ${ }^{19} \mathrm{~F}-\mathrm{NMR}$ resonance at $\delta=-55 \mathrm{ppm}$. For a model with $2 \mathrm{Ge}$ atoms per $\left[4 \cdot 5^{6}\right]$ cage, an incorporation of fluoride in $\left[4 \cdot 5^{6}\right]_{2 G e, p a i r}$ cages is actually even favoured over the $d 4 r_{0 G e}$ cages. Moving towards models with $d 4 r_{1 G e}$ and $d 4 r_{2 G e, p a i r}$ cages, it is always more favourable to retain fluoride in the 
$d 4 r$ cages than in the $\left[4 \cdot 5^{6}\right]$ cages, and the evolution of the $\Delta E\left(F @ d 4 r-F @\left[4 \cdot 5^{6}\right]\right)$ values shows that an increase in the number of $\mathrm{Ge}$ atoms per cage tends to further stabilise the $d 4 r$ building units. The high stability of these fluoride environments agrees with the negligible removal from $d 4 r$ units in Gecontaining ITQ-13 samples with high and intermediate Si/Ge ratios. ${ }^{[11]}$

Table 5: Stability difference between partially defluorinated ITQ-13 models containing fluoride only in $d 4 r$ cages or only in $\left[4 \cdot 5^{6}\right]$ cages. The first two columns give the number of Ge atoms in the different cages.

\begin{tabular}{llc}
\hline \hline$d 4 r$ cage & {$\left[4 \cdot 5^{6}\right]$ cage } & $\begin{array}{c}\Delta E\left(\mathrm{~F} @ d 4 r-\mathrm{F} @\left[4 \cdot 5^{6}\right]\right) \\
/ \mathrm{kJ} \mathrm{mol}^{-1}\end{array}$ \\
\hline$d 4 r_{0 \mathrm{Ge}}$ & {$\left[4 \cdot 5^{6}\right]_{0 \mathrm{Ge}}$} & -50.4 \\
$d 4 r_{0 \mathrm{Ge}}$ & {$\left[4 \cdot 5^{6}\right]_{1 \mathrm{Ge}}$} & -7.2 \\
$d 4 r_{0 \mathrm{Ge}}$ & {$\left[4 \cdot 5^{6}\right]_{2 \mathrm{Ge}, \text { pair }}$} & 10.0 \\
$d 4 r_{1 \mathrm{Ge}}$ & {$\left[4 \cdot 5^{6}\right]_{0 \mathrm{Ge}}$} & -71.5 \\
$d 4 r_{1 \mathrm{Ge}}$ & {$\left[4 \cdot 5^{6}\right]_{1 \mathrm{Ge}}$} & -34.2 \\
$d 4 r_{1 \mathrm{Ge}}$ & {$\left[4 \cdot 5^{6}\right]_{2 \mathrm{Ge} \text { pair }}$} & -16.4 \\
$d 4 r_{2 \mathrm{Ge}, \mathrm{pair}}$ & {$\left[4 \cdot 5^{6}\right]_{0 \mathrm{Ge}}$} & -95.2 \\
$d 4 r_{2 \mathrm{Ge}, \mathrm{pair}}$ & {$\left[4 \cdot 5^{6}\right]_{1 \mathrm{Ge}}$} & -55.8 \\
$d 4 r_{2 \mathrm{Ge}, \text { pair }}$ & {$\left[4 \cdot 5^{6}\right]_{2 \mathrm{Ge}, \mathrm{pair}}$} & -37.0 \\
\hline \hline
\end{tabular}

\section{Conclusions}

DFT calculations considering one Ge atom per ITQ-13 unit cell reproduce the experimentally observed preference for the $\mathrm{T} 2, \mathrm{~T} 5$, and $\mathrm{T} 7$ sites only if the OSDA cations and fluoride anions are included. Upon increasing the number of Ge atoms per cell, purely siliceous $d 4 r$ cages and $\left[4 \cdot 5^{6}\right]$ cages disappear quickly. This is ascribed to a strong energetic preference for a distribution of Ge among the available building units, as opposed to a coexistence of purely siliceous cages and cages with several Ge atoms at the corners. At higher Ge contents, a range of local environments are expected to coexist. These results are in accord with both the large width of the ${ }^{19} \mathrm{~F}-\mathrm{NMR}$ resonances observed at low Si/Ge ratios, and with the presence of the $\delta=-8,-20 \mathrm{ppm}$, and $-55 \mathrm{ppm}$ resonances at $\mathrm{Si} / \mathrm{Ge}$ ratios as low as 3. It is not unusual to observe a considerable scatter in the total energies obtained with different models containing the same type of building units, indicating that factors other than the local environment play a role.

Whereas experimental evidence for $\left[4^{\cdot} 5^{6}\right]_{1 G e}$ cages with one Ge atom in the basal 4MR plane had already been presented, the calculations predict $\left[4 \cdot 5^{6}\right]_{2 G e \text {,pair }}$ cages to be stable at intermediate and low $\mathrm{Si} / \mathrm{Ge}$ ratios. As the calculated ${ }^{19} \mathrm{~F}$ chemical shift falls between the strong and broad $\delta=-8 \mathrm{ppm}$ and - 
20 ppm peaks, the contribution of these cages may have been overlooked. Further experimental work using advanced NMR methods is thus needed to clarify this point. We note that an occupation of not more than two T sites per $4 \mathrm{MR}$ means that the $\left[4 \cdot 5^{6}\right]$ and $\left[4 \cdot 5^{2} \cdot 6^{2}\right]$ cages would stay connected via two shared T sites if the Ge atoms were removed. Hence, the computational prediction of $\left[4 \cdot 5^{6}\right]_{2 G e, p a i r}$ cages does not disagree with the observation that the layers oriented perpendicular to the $a$ axis remain intact upon degermanation of Ge-rich ITQ-13 samples. ${ }^{[13]}$

The AIMD simulations do not indicate that the fluoride anions in the $\left[4 \cdot 5^{6}\right]$ cages are dynamically disordered at room temperature. Thus, the experimentally observed disorder in the crystal structure is of a static nature, in accordance with the presence of a (weak) resonance at $\delta=-146 \mathrm{ppm}$ in the ${ }^{19} \mathrm{~F}$ to ${ }^{29} \mathrm{Si}$ cross-polarisation NMR spectrum. ${ }^{[4]}$ At $408 \mathrm{~K}$, a typical zeolite synthesis temperature, the dynamic behaviour of fluoride anions strongly depends on the local environment: Whereas dynamic "jumps" between different Si atoms occur in purely siliceous cages, displacements away from the Ge atom are short-lived in $\left[4 \cdot 5^{6}\right]_{1 \mathrm{Ge}}$ cages. If there are two Ge atoms at adjacent corners, the fluoride anions move rapidly back and forth between them. It is of interest to assess whether NMR is able to probe these distinct scenarios, potentially leading to further structural insights regarding the occurrence of different types of $\left[4 \cdot 5^{6}\right]$ cages. For fluoride in $d 4 r$ cages, the observed trends agree with those of a previous AIMD study of AST-type silicogermanates, ${ }^{[27]}$ indicating that the local structure is the main factor determining the freedom of motion.

Regarding partially defluorinated models, the calculations provide clear evidence for the exceptional stability of fluoride in $d 4 r$ cages, both for all-silica and Ge-containing ITQ-13. This observation agrees with the experimental finding of a selective defluorination of $\left[4 \cdot 5^{6}\right]_{0 G e}$ and $\left[4 \cdot 5^{6}\right]_{1 G e}$ cages upon alkaline treatment, and indicates that the higher thermodynamic stability of fluoride anions in $d 4 r$ cages is a key factor preventing their removal, despite the rather harsh treatment conditions (however, it cannot be ruled out that kinetic effects may also play a role in the more facile removal of fluoride from $\left[4 \cdot 5^{6}\right]$ cages). Although the calculations indicate that $\left[4 \cdot 5^{6}\right]_{2 G e, p a i r}$ cages provide an even more stable fluoride environment than $d 4 r_{0 G e}$ cages, it is likely that these building units do not coexist in real ITQ-13 samples because $\left[4 \cdot 5^{6}\right]$ cages with more than one Ge atom will appear only at relatively high Ge contents. Admittedly, the DFT results do not explain the near-complete defluorination of Ge-rich samples. This behaviour may be due to a temporary breaking of $\mathrm{Ge}-\mathrm{O}-\mathrm{Ge}$ linkages forming several adjacent edges of the $d 4 r$ units, especially when there are four Ge atoms forming an entire $4 \mathrm{MR}\left(d 4 r_{4 G e, 4 G e-0-G e}\right.$, Figure $3 \mathbf{d}$ ). An approach based solely on DFT optimisations of partially defluorinated models cannot capture the possibility of a temporary bond breaking. Therefore, future work will employ AIMD simulations to investigate the interaction of ITQ-13 with alkaline or acid media. 


\section{Supporting Information}

Supporting Information (PDF format) contains details of individual ITQ-13 models as well as full results of DFT optimisations, visualisation of low-energy structures, tables and figures reporting individual RMSD values, and full results for partially defluorinated systems. Sample input files, DFT-optimised structures of ITQ-13 silicogermanates (PDB format), and AIMD trajectories (PDB format) have been deposited in the Figshare repository at https://doi.org/10.6084/m9.figshare.17067752.

\section{Acknowledgment}

M.F. acknowledges funding by the Deutsche Forschungsgemeinschaft (DFG, German Research Foundation), project number 389577027 (FI 1800/5-1). Calculations were performed with resources provided by the North-German Supercomputing Alliance (HLRN), project hbc00030 (Dynamics of fluoride anions in all-silica and silicogermanate zeolites). C.B. acknowledges FCT for Doctoral Fellowship PD/BD/142849/2018 integrated in the Ph.D. program in NMR applied to chemistry, materials, and biosciences (Grant PD/00065/2013). This work was developed within the scope of the project CICECO-Aveiro Institute of Materials, Grants UIDB/50011/2020 and UIDP/50011/2020, financed by national funds through the FCT/MEC and when appropriate cofinanced by FEDER under the PT2020 Partnership Agreement.

\section{References}

[1] T. Boix, M. Puche, M. A. Camblor, A. Corma, Synthetic Porous Crystalline Material ITQ-13, Its Synthesis and Use, 2002, US Patent 6471941B1.

[2] A. Corma, M. Puche, F. Rey, G. Sankar, S. J. Teat, Angew. Chemie Int. Ed. 2003, 42, 1156-1159.

[3] R. Castañeda, A. Corma, V. Fornés, J. Martínez-Triguero, S. Valencia, J. Catal. 2006, 238, $79-87$.

[4] J. A. Vidal-Moya, T. Blasco, F. Rey, A. Corma, M. Puche, Chem. Mater. 2003, 15, 3961-3963.

[5] A. I. Hussain, A. Palani, A. M. Aitani, J. Čejka, M. Shamzhy, M. Kubů, S. S. Al-Khattaf, Fuel Process. Technol. 2017, 161, 23-32.

[6] W. Skistad, S. Teketel, F. L. Bleken, P. Beato, S. Bordiga, M. H. Nilsen, U. Olsbye, S. Svelle, K. P. Lillerud, Top. Catal. 2014, 57, 143-158.

[7] C. Baerlocher, L. B. McCusker, http://www.iza-structure.org/databases/, 2021.

[8] N. A. Anurova, V. A. Blatov, G. D. Ilyushin, D. M. Proserpio, J. Phys. Chem. C 2010, 114, 10160-10170.

[9] A. Pulido, A. Corma, G. Sastre, J. Phys. Chem. B 2006, 110, 23951-23961. 
[10] X. Liu, U. Ravon, A. Tuel, Angew. Chemie Int. Ed. 2011, 50, 5900-5903.

[11] X. Liu, U. Ravon, A. Tuel, Chem. Mater. 2011, 23, 5052-5057.

[12] X. Liu, Y. Chu, Q. Wang, W. Wang, C. Wang, J. Xu, F. Deng, Solid State Nucl. Magn. Reson. 2017, 87, 1-9.

[13] M. Shamzhy, M. Opanasenko, Y. Tian, K. Konysheva, O. Shvets, R. E. Morris, J. Čejka, Chem. Mater. 2014, 26, 5789-5798.

[14] P. Lu, L. Gómez-Hortigüela, M. A. Camblor, Chem. - A Eur. J. 2019, 25, 1561-1572.

[15] P. Caullet, J. L. Guth, J. Hazm, J. M. Lamblin, H. Gies, Eur. J. Solid State Inorg. Chem. 1991, 28, 345-361.

[16] H. Koller, A. Wölker, L. A. Villaescusa, M. J. Diaz-Cabañas, S. Valencia, M. A. Camblor, J. Am. Chem. Soc. 1999, 121, 3368-3376.

[17] G. Sastre, A. Pulido, A. Corma, Chem. Commun. 2005, 2357-2359.

[18] A. Pulido, G. Sastre, A. Corma, ChemPhysChem 2006, 7, 1092-1099.

[19] R. T. Rigo, S. R. G. Balestra, S. Hamad, R. Bueno-Perez, A. R. Ruiz-Salvador, S. Calero, M. A. Camblor, J. Mater. Chem. A 2018, 6, 15110-15122.

[20] M. V. Shamzhy, M. V. Opanasenko, F. S. D. O. Ramos, L. Brabec, M. Horáček, M. Navarro-Rojas, R. E. Morris, H. D. O. Pastore, J. Čejka, Catal. Sci. Technol. 2015, 5, 2973-2984.

[21] V. I. Kasneryk, M. V. Shamzhy, M. V. Opanasenko, J. Čejka, J. Energy Chem. 2016, 25, 318-326.

[22] G. Sastre, A. Pulido, R. Castañeda, A. Corma, J. Phys. Chem. B 2004, 108, 8830-8835.

[23] G. Sastre, A. Pulido, A. Corma, Microporous Mesoporous Mater. 2005, 82, 159-163.

[24] P. Kamakoti, T. A. Barckholtz, J. Phys. Chem. C 2007, 111, 3575-3583.

[25] P. St. Petkov, H. A. Aleksandrov, V. Valtchev, G. N. Vayssilov, Chem. Mater. 2012, 24, 2509-2518.

[26] S. O. Odoh, M. W. Deem, L. Gagliardi, J. Phys. Chem. C 2014, 118, 26939-26946.

[27] M. Fischer, J. Phys. Chem. C 2019, 123, 1852-1865.

[28] E. El Hayek, B. Harbuzaru, J. A. Martens, C. Chizallet, Microporous Mesoporous Mater. 2020, 306, 110425.

[29] T. D. Kühne, M. Iannuzzi, M. Del Ben, V. V. Rybkin, P. Seewald, F. Stein, T. Laino, R. Z. Khaliullin, O. Schütt, F. Schiffmann, D. Golze, J. Wilhelm, S. Chulkov, M. H. Bani-Hashemian, V. Weber, U. Borštnik, M. Taillefumier, A. S. Jakobovits, A. Lazzaro, H. Pabst, T. Müller, R. Schade, M. Guidon, S. Andermatt, N. Holmberg, G. K. Schenter, A. Hehn, A. Bussy, F. Belleflamme, G. Tabacchi, A. Glöß, M. Lass, I. Bethune, C. J. Mundy, C. Plessl, M. Watkins, J. VandeVondele, M. Krack, J. Hutter, J. Chem. Phys. 2020, 152, 194103.

[30] J. VandeVondele, M. Krack, F. Mohamed, M. Parrinello, T. Chassaing, J. Hutter, Comput. Phys. Commun. 2005, 167, 103-128.

[31] J. P. Perdew, K. Burke, M. Ernzerhof, Phys. Rev. Lett. 1996, 77, 3865-3868.

[32] S. Grimme, J. Antony, S. Ehrlich, H. Krieg, J. Chem. Phys. 2010, 132, 154104. 
[33] J. VandeVondele, J. Hutter, J. Chem. Phys. 2007, 127, 114105.

[34] M. Krack, Theor. Chem. Acc. 2005, 114, 145-152.

[35] S. Nosé, J. Chem. Phys. 1984, 81, 511-519.

[36] W. G. Hoover, Phys. Rev. A 1985, 31, 1695-1697.

[37] W. Humphrey, A. Dalke, K. Schulten, J. Mol. Graph. 1996, 14, 33-38.

[38] K. Momma, F. Izumi, J. Appl. Crystallogr. 2011, 44, 1272-1276.

[39] K. Wolinski, J. F. Hinton, P. Pulay, J. Am. Chem. Soc. 1990, 112, 8251-8260.

[40] M. J. Frisch, G. W. Trucks, H. B. Schlegel, G. E. Scuseria, M. A. Robb, J. R. Cheeseman, G. Scalmani, V. Barone, G. A. Petersson, H. Nakatsuji, X. Li, M. Caricato, A. V Marenich, J. Bloino, B. G. Janesko, R. Gomperts, B. Mennucci, H. P. Hratchian, J. V Ortiz, A. F. Izmaylov, J. L. Sonnenberg, D. Williams-Young, F. Ding, F. Lipparini, F. Egidi, J. Goings, B. Peng, A. Petrone, T. Henderson, D. Ranasinghe, V. G. Zakrzewski, J. Gao, N. Rega, G. Zheng, W. Liang, M. Hada, M. Ehara, K. Toyota, R. Fukuda, J. Hasegawa, M. Ishida, T. Nakajima, Y. Honda, O. Kitao, H. Nakai, T. Vreven, K. Throssell, J. A. Montgomery Jr., J. E. Peralta, F. Ogliaro, M. J. Bearpark, J. J. Heyd, E. N. Brothers, K. N. Kudin, V. N. Staroverov, T. A. Keith, R. Kobayashi, J. Normand, K. Raghavachari, A. P. Rendell, J. C. Burant, S. S. Iyengar, J. Tomasi, M. Cossi, J. M. Millam, M. Klene, C. Adamo, R. Cammi, J. W. Ochterski, R. L. Martin, K. Morokuma, O. Farkas, J. B. Foresman, D. J. Fox, Gaussian 16 Revision C.01, Gaussian, Inc., Wallingford CT, 2016, 2016.

[41] C. Adamo, V. Barone, J. Chem. Phys. 1999, 110, 6158-6170.

[42] A. D. Becke, Phys. Rev. A 1988, 38, 3098-3100.

[43] A. D. Becke, J. Chem. Phys. 1993, 98, 5648-5652.

[44] T. H. Dunning, J. Chem. Phys. 1989, 90, 1007-1023.

[45] E. R. Davidson, Chem. Phys. Lett. 1996, 260, 514-518.

[46] S. V. Fedorov, L. B. Krivdin, J. Fluor. Chem. 2020, 238, 109625.

[47] M. Fischer, W. J. Kim, M. Badawi, S. Lebègue, J. Chem. Phys. 2019, 150, 094102.

[48] M. Fischer, J. Phys. Chem. C 2021, 125, 8825-8839.

[49] M. P. Attfield, C. R. A. Catlow, A. A. Sokol, Chem. Mater. 2001, 13, 4708-4713.

[50] D. S. Wragg, R. E. Morris, A. W. Burton, Chem. Mater. 2008, 20, 1561-1570.

[51] B. Cordero, V. Gómez, A. E. Platero-Prats, M. Revés, J. Echeverría, E. Cremades, F. Barragán, S. Alvarez, Dalton Trans. 2008, 2832-2838.

[52] R. D. Shannon, Acta Crystallogr. Sect. A 1976, 32, 751-767.

[53] K. Okhotnikov, T. Charpentier, S. Cadars, J. Cheminform. 2016, 8, 17.

[54] H. Koller, A. Wölker, H. Eckert, C. Panz, P. Behrens, Angew. Chemie Int. Ed. 1997, 36, 2823-2825.

[55] M. Fischer, J. Phys. Chem. C 2020, 124, 5690-5701.

[56] H. B. Bürgi, S. C. Capelli, H. Birkedal, Acta Crystallogr. Sect. A Found. Crystallogr. 2000, 56, 425-435. 